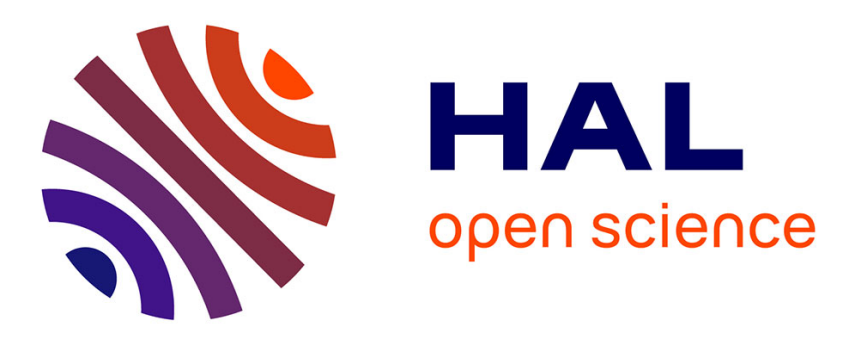

\title{
A penalty-based multifiber finite element model for coupled bending and torsional-warping analysis of composite beams
}

\author{
M Lezgy-Nazargah, Philippe Vidal, Olivier Polit
}

\section{- To cite this version:}

M Lezgy-Nazargah, Philippe Vidal, Olivier Polit. A penalty-based multifiber finite element model for coupled bending and torsional-warping analysis of composite beams. European Journal of Mechanics - A/Solids, 2020, 80 (Article 103915), 10.1016/j.euromechsol.2019.103915 . hal-03420979

\section{HAL Id: hal-03420979 \\ https://hal.parisnanterre.fr/hal-03420979}

Submitted on 9 Nov 2021

HAL is a multi-disciplinary open access archive for the deposit and dissemination of scientific research documents, whether they are published or not. The documents may come from teaching and research institutions in France or abroad, or from public or private research centers.
L'archive ouverte pluridisciplinaire HAL, est destinée au dépôt et à la diffusion de documents scientifiques de niveau recherche, publiés ou non, émanant des établissements d'enseignement et de recherche français ou étrangers, des laboratoires publics ou privés. 


\title{
A penalty-based multifiber finite element model for coupled bending and torsional-warping analysis of composite beams
}

\author{
M. Lezgy-Nazargah ${ }^{\mathrm{a}, *}, \mathrm{P}$. Vidal $^{\mathrm{b}}$, O. Polit ${ }^{\mathrm{b}}$ \\ ${ }^{\text {a }}$ Faculty of Civil Engineering, Hakim Sabzevari University, Sabzevar, 9617976487-397, Iran \\ ${ }^{\mathrm{b}}$ LEME, UPL, Univ Paris Nanterre, 50 rue de Sevres, 92410, Ville d'Avray, France
}

\section{Introduction}

Laminated composite and sandwich beams are extensively used in various manufacturing fields as main structural components. They have special advantages like high stiffness to mass ratio as well as superior strength to weight ratio. Development of efficient mathematical models for the estimation of structural responses of composite beams is a key issue.

The composite beams are usually subjected to arbitrarily bending and/or torsional loads. Various laminated beam theories were introduced in the last two decades for modeling the pure bending behavior of composite and sandwich beams (e.g., layer-wise theories (Carrera, 2000; Reddy, 2004), zigzag theories (Icardi, 2001a, 2001b; Carrera, 2003; Katariya and Panda, 2019; Katariya et al., 2019), and global-local theories (Shariyat, 2010; Lezgy-Nazargah et al., 2011; Lezgy-Nazargah, 2017; Beheshti-Aval and Lezgy-Nazargah, 2012)). On the other hand, torsion of beams has been a classical subject in solid mechanics. Coulomb proposed a solution for the approximation of torsional resistance of circular bars (Wunderlich and Pilkey, 2003). Coulomb's formulation is based on the assumption that the cross-section of bars stay plane after torsion. By making assumptions on the deformations and using the semi-inverse method, Saint-Venant introduced an accurate solution for the torsion of bars (Timoshenko and Goodier, 1970). The assumed deformations of Saint-Venant include an unknown warping function. He computed this unknown warping function from the satisfaction of the equilibrium conditions as well as the free-conditions of stresses on surface boundaries. According to the Saint-Venant theory, the torsional stresses only take place if warping is unrestrained. The aforementioned condition is very rare in engineering practice. Indeed, warping is usually restrained at supports, at the location of imposed torsion moments and where the thickness of cross-section changes. To overcome the limitations of Saint-Venant's theory, "restrained warping theory" or "non-uniform torsion theory" was developed by Vlasov (1959).

By using Prandtl's stress function, Savoia and Tullini (1993) introduced a formulation for the prediction of torsional-warping responses of composite beams with arbitrary cross-section. Based on eigenfunction expansion method, Swanson (1998) derived an exact solution for the response of rectangular laminated orthotropic beams under uniform torsion. Saygun et al. (2007) presented a 2D finite element model for calculation of torsional rigidity of the composite beams. They first derived the differential equation by using the Saint-Venant's stress

\footnotetext{
* Corresponding author.

E-mail addresses: m.lezgy@hsu.ac.ir (M. Lezgy-Nazargah), philippe.vidal@parisnanterre.fr (P. Vidal), olivier.polit@parisnanterre.fr (O. Polit).
} 
function. Then, they obtained discretized form of partial differential equations by means of the finite element method. By including the effects of shear forces and torsion in the formulations, El Fatmi (El Fatmi, 2007) introduced a beam theory with a non-uniform warping. The beam theory of El Fatmi (El Fatmi, 2007) is valid for any homogeneous cross-section made of isotropic elastic material. El Fatmi's beam theory is based on a kinematic which includes six independent unknown warping parameters and nine independent warping functions. Starting from this kinematic and using the principle of minimum potential energy, El Fatmi derived his beam theory. Extension of El Fatmi's beam theory for homogeneous and composite cross-sections is carried out in El Fatmi and Zenzri (El Fatmi and Zenzri, 2004). Based on anisotropic theory of elasticity and following the Sokolnikoff's approach, Demakos (2003) determined stress and displacement components in the cantilever fibre-reinforced composite laminate beams subjected to combined action of bending and torsion moments. El Fatmi and Ghazouani (El Fatmi and Ghazouani, 2011) proposed a high-order theory for analysis of composite beams under the action of bending/torsion loads. They modified Saint-Venant theory so that it could be used for the prediction of 3D stresses in the regions near the end-sections of beams. Ganapathi et al. (1999) introduced a finite element model for the analysis of rectangular sandwich beams considering warping and transverse shear effects. They obtained warping function of sandwich beam from a 3D elasticity solution. Santiuste et al. (2008) studied the dynamic behavior of laminated beams by using Flexibility Influence Function Method. The 1D model of Santiuste and colleagues takes into account the effects of rotatory inertia, shear force, and bending-torsion coupling.

In this paper an efficient numerical tool is developed for quasi-3D analysis of laminated composite and sandwich beams under combined transversal and torsional loading. Thus, a new multifiber finite element model with low degrees of freedom (dofs) is introduced. A displacement field which includes seven generalized displacement unknown parameters and a $2 \mathrm{D}$ unknown warping function is assumed for representing the kinematic of beam. For both displacement components and transverse shear stresses, all continuity conditions are satisfied at the layer interfaces. Traction-free requirements of stresses on the boundary surfaces of beam are also fulfilled in the present formulation. Thanks to the use of Cosine function for representing the transverse shear deformations due to bending, the shear correction factor is not needed in the presented model. A 2D penalty-based finite element model has been developed to determine the unknown warping function of beam. A 1D three-node multifiber finite element is employed for the computation of generalized displacement unknown parameters of beam. For validation, results of the present model have been compared with the results obtained from 3D finite element simulation (ABAQUS), and numerical and analytical results reported by other researchers. These comparisons show that the present model can accurately predict the coupled bending and torsional-warping responses of laminated composite and sandwich beams by using small number of elements and degrees of freedom.

This paper is organized as follows. Constitutive equations as well as kinematic relations of composite beams are introduced in section 2 . Penalty-based finite element model employed for the computation of restrained warping function of composite beams is described in Section 3. Three-node 1D multifiber finite element developed for the approximation of unknown variables of displacement field is introduced in Section 4. Validation examples and discussion on numerical results are given in Section 5. Conclusions and comments for the future researches are presented in Section 6.

\section{Basic equations}

\subsection{Geometry and coordinate system}

As depicted in Fig. 1, a straight composite beam with a rectangular cross-section is considered in the present study. It is made of $N C$ layers with different material properties. The material of each layer is assumed to be homogeneous, linearly elastic and transversely isotropic in the beam axes. As depicted in Fig. 1, the rectangular Cartesian coordinate system $(x, y, z)$ is considered for studying the structural behavior of composite beams. The $x$-axis is along the length, $y$-axis is along the width and $z$-axis is along the height of beam. $y$ - and $z$-axis intersect each other at the centroid of the composite cross-section, i.e. point "O".

\subsection{Constitutive law}

By neglecting the effects of transverse normal stresses $\left(\sigma_{y y}^{k} \quad \sigma_{z z}^{k}\right.$ 0 ), the constitutive law for the kth layer of beam can be expressed as:

$\overline{\boldsymbol{\sigma}}^{k} \quad \overline{\mathbf{C}}^{k} \overline{\boldsymbol{\varepsilon}}^{k}$

where

$\overline{\boldsymbol{\sigma}}^{k} \quad\left[\begin{array}{llll}\sigma_{x x}^{k} & \sigma_{y z}^{k} & \sigma_{x z}^{k} & \sigma_{x y}^{k}\end{array}\right]^{T}$

$\overline{\boldsymbol{\varepsilon}}^{k} \quad\left[\begin{array}{cccc}\varepsilon_{x x}^{k} & 2 \varepsilon_{y z}^{k} & 2 \varepsilon_{x z}^{k} & 2 \varepsilon_{x y}^{k}\end{array}\right]^{T}$

$\overline{\mathbf{C}}^{k}\left[\begin{array}{cccc}C_{11}^{k} & 0 & 0 & C_{16}^{k} \\ 0 & C_{44}^{k} & C_{45}^{k} & 0 \\ 0 & C_{54}^{k} & C_{55}^{k} & 0 \\ C_{61}^{k} & 0 & 0 & C_{66}^{k}\end{array}\right]$

$\left[\begin{array}{cc}C_{11}^{k} & C_{13}^{k} \\ 0 & 0 \\ 0 & 0 \\ C_{62}^{k} & C_{63}^{k}\end{array}\right]\left[\begin{array}{ll}C_{22}^{k} & C_{23}^{k} \\ C_{32}^{k} & C_{33}^{k}\end{array}\right]^{1}\left[\begin{array}{llll}C_{21}^{k} & 0 & 0 & C_{26}^{k} \\ C_{31}^{k} & 0 & 0 & C_{36}^{k}\end{array}\right]$
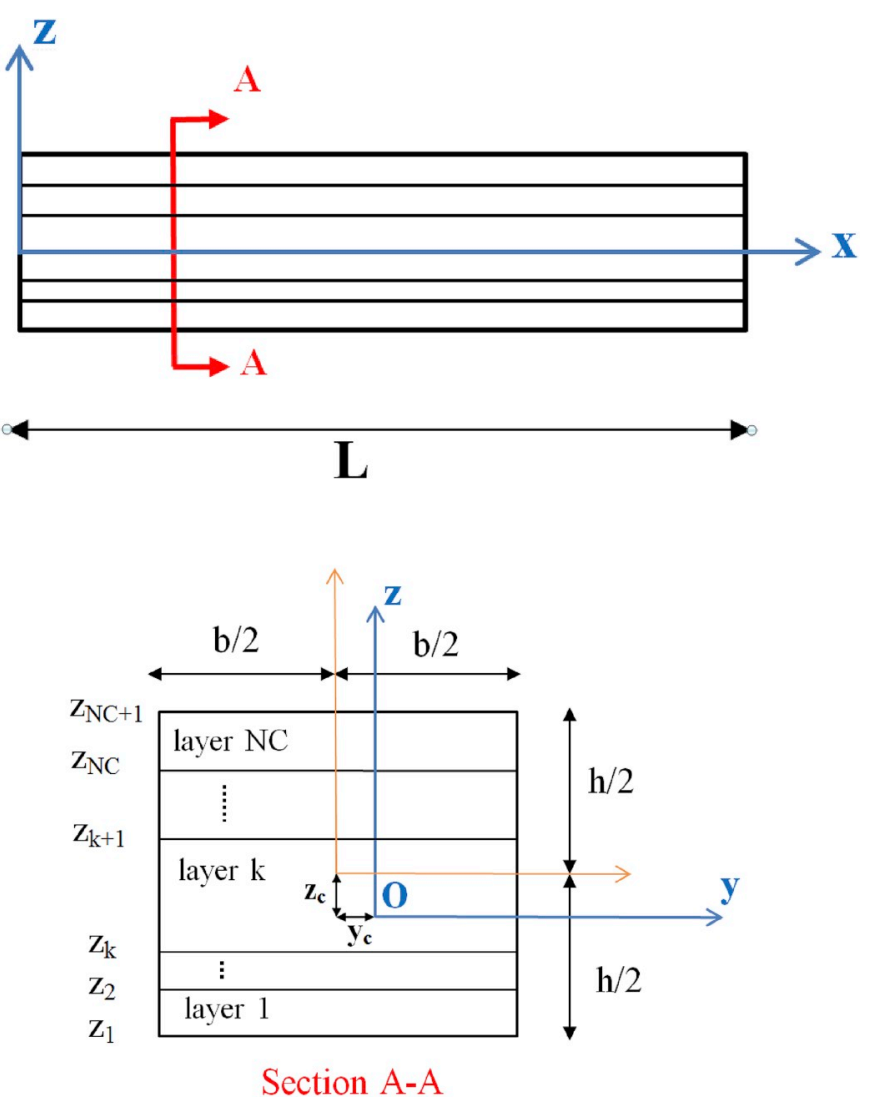

Fig. 1. The composite beam and Cartesian coordinate system. 
and $C_{i j}, \sigma_{i j}$ and $\varepsilon_{i j}$ denote the 3D elastic material constants, stress, and strain components, respectively.

\subsection{Kinematic descriptions}

2.3.1. Torsional-warping around $x$-axis.Displacement components corresponding to the kth layer at an arbitrary point $P(x, y, z)$ due to torsion and warping can be expressed as

$U_{t}^{k} x, y, z \quad \bar{\varphi}^{k} y, z \gamma x$

$V_{t}^{k} x, y, z \quad z \quad z_{t} \theta_{x} x$

$W_{t}^{k} x, y, z \quad y \quad y_{t} \theta_{x} x$

where $U_{t}^{k}, V_{t}^{k}$ and $W_{t}^{k}$ are the displacement components of composite beam due to torsion and warping along $x, y$ and $z$ directions, respectively. $\bar{\varphi}^{k} y, z$ is the restrained warping function which describes the distortion of beam in the longitudinal direction due to warping. $\gamma$ is an unknown parameter which represents the intensity of torsional rotation gradient in the length direction. The torsional rotation of the crosssection around the $x$-axis is represented by $\theta_{x} x$. Finally, $y_{t}, z_{t}$ represents the position of twist center with respect to the Coordinate system $(x, y, z)$. In the Saint-Venant's theory of torsion, which is valid only for constant warping and no restraints at the ends, it is proved that the cross-section twists about the centroid. In case of restrained torsion, the cross-section twists about twist center. Twist or shear center is a fixed point in the cross-section which does not move due to torsion. By using the transformation of coordinates, $\bar{\varphi} y, z$ can be defined with respect to unrestrained warping function $\varphi y, z$ as below (Connor, 1976):

$\bar{\varphi} y, z \quad \frac{1}{\Omega} \int_{\Omega} \varphi y, z d \Omega \quad z_{t} y \quad y_{t} z \quad \varphi y, z$

where $\Omega$ denotes the total area of cross-section. The location of the twist center can be calculated from the following relations (Connor, 1976):

$y_{t} \quad \int_{\Omega} z \varphi \quad y, z d \Omega \quad \int_{\Omega} z^{2} d \Omega$

$z_{t} \quad \int_{\Omega} y \varphi \quad y, z d \Omega \quad \int_{\Omega} y^{2} d \Omega$

Eq. (2) can be written as the following matrix form:

$\Lambda_{t}^{k} \quad \mathbf{O}_{t}^{k} \mathbf{u}_{t}$

where $\Lambda_{t}^{k} \quad U_{t}^{k} \quad V_{t}^{k} \quad W_{t}^{k}{ }^{T}, \mathbf{u}_{\mathbf{t}} \quad\left\{\begin{array}{ll}\theta_{x} & \gamma\end{array}\right\}^{T}$ and

$\mathbf{O}_{t}{ }^{k} \quad\left[\begin{array}{ccc}0 & \bar{\varphi}^{k} y, z \\ z & z_{t} & 0 \\ y & y_{t} & 0\end{array}\right]$

Based on well-known displacement-strain relations, the following matrix expression are obtained for the strain components due to torsion and warping of composite beam:

$\left\{\begin{array}{c}\varepsilon_{x x}^{t k} \\ 2 \varepsilon_{y z}^{t k} \\ 2 \varepsilon_{x z}^{t k} \\ 2 \varepsilon_{x y}^{t k}\end{array}\right\} \quad\left[\begin{array}{cc}0 & \varphi^{k} \frac{d}{d x} \\ 0 & 0 \\ y \frac{d}{d x} & \frac{\partial \varphi^{k}}{\partial z} \\ z \frac{d}{d x} & \frac{\partial \varphi^{k}}{\partial y}\end{array}\right] \begin{gathered}\theta_{x} \\ \gamma\end{gathered}$

or

$\overline{\boldsymbol{\varepsilon}}_{t}^{k} \quad \mathbf{B}_{t}^{k} \mathbf{u}_{t}$
In Eq. (6.a), the superscript " $t$ " represents the torsional-warping contribution of strain components. Note that the torsional-warping contribution of axial displacement and transverse shear stress in $x-z$ plane must be continuous at the interface between layers. In section 3, warping function $\varphi^{k} y, z$ will be determined by enforcing the continuity conditions of $U_{t}^{k}$ and $\sigma_{x z}^{t k}$ at the interface between layers.

\subsubsection{Axial extension and bending in $x-y$ plane}

Displacement components due to axial extension as well as bending in $x$-y plane can be expressed as:

$U_{b 2}^{k} x, y, z \quad u x \quad y \quad y_{c} \frac{d v x}{d x} \quad \theta_{z} x \quad \frac{d v x}{d x} f y$

$V_{b 2}^{k} x, y, z \quad v x$

$W_{b 2}^{k} x, y, z \quad 0$

where $f y \quad \frac{b}{\pi} \sin \frac{\pi y y_{c}}{b}$ and sub-function $u x$ represents the uniform extension of cross-section of the beam along $x$-axis. $v x$ is bending deflection along $y$ direction, and $\theta_{z} x$ is independent shear bending rotation around the $z$ axis. The matrix form of Eq. (7) is given by:

$\mathbf{\Lambda}_{b 2}^{k} \quad \mathbf{O}_{b 2}^{k} \mathbf{u}_{b 2}$

where $\Lambda_{b 2}^{k} \quad U_{b 2}^{k} \quad V_{b 2}^{k} \quad W_{b 2}^{k}{ }^{T}, \mathbf{u}_{b 2} \quad\left\{\begin{array}{lll}u & v & \theta_{z}\end{array}\right\}^{T}$ and

$\mathbf{O}_{b 2}^{k}\left[\begin{array}{cccccc}1 & y_{c} & y & f y & \frac{d}{d x} & f y \\ 0 & 1 & & 0 \\ 0 & 0 & & 0\end{array}\right]$

Strain components due to uniform extension and bending in $x-y$ plane can be written as below:

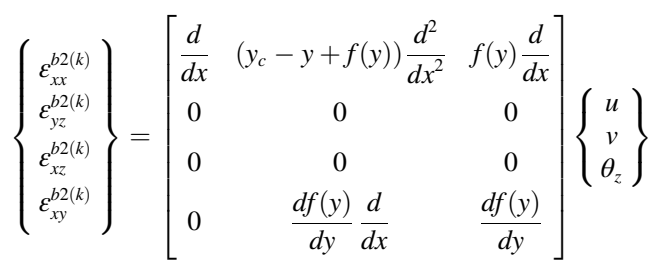

or

$\overline{\mathbf{\varepsilon}}_{b 2}^{k} \quad \mathbf{B}_{b 2}^{k} \mathbf{u}_{b 2}$

Thanks to representing the transverse shear strain by using a cosine function, it is obvious that the free traction requirements of transverse shear stress on the planes $y \quad b / 2 \quad y_{t}$ and $y \quad b / 2 \quad y_{t}$ is satisfied. Thus, no shear correction factor is needed for the bending in $x-y$ plane.

\subsubsection{Bending in $x-z$ plane}

Based on refined sinus beam theory (Vidal and Polit, 2008; Beheshti-Aval et al., 2011; Lezgy-Nazargah et al., 2015), pure bending in $x-z$ plane can be defined by the following relations:

$U_{b 3}^{k} x, y, z \quad z \quad z_{c} \frac{d w x}{d x} \quad \theta_{y} x \quad \frac{d w x}{d x} F z \quad u_{l o c} x S z$

$V_{b 3}^{k} x, y, z \quad 0$

$W_{b 3}^{k} x, y, z \quad w x$

$w x$ denotes bending deflection along $z$ direction. $\theta_{y} x$ is independent shear bending rotation around the $y$ axis, and $u_{l o c} x$ is a local unknown parameter related to the first layer. The functions $F z$ and $S z$ are given below: 


$$
\begin{gathered}
\left.\left.\left.\left.F \quad z) \frac{h}{\pi} \sin \frac{\pi z}{h}\right) \sum_{k=1}^{N C} \xi_{k} \beta_{1}^{k} \quad \frac{1}{2} \frac{3 \xi_{k}^{2}}{2}\right) \beta_{2}^{k} \quad \frac{3 \xi_{k}}{2} \frac{5 \xi_{k}^{3}}{2}\right) \beta_{3}^{k}\right) \\
\left.\left.\left.\begin{array}{lllll}
H & z & z_{k}
\end{array}\right) \quad \begin{array}{lllll}
H & z & z_{k} & 1
\end{array}\right)\right)
\end{gathered}
$$$$
\begin{gathered}
\left.S \quad z) \sum_{k=1}^{N C} \xi_{k} \delta_{1}^{k} \quad \frac{1}{2} \frac{3 \xi_{k}^{2}}{2}\right) \delta_{2}^{k} \\
\left.\left.\begin{array}{llll}
H & z & z_{k} & 1
\end{array}\right)\right)
\end{gathered}
$$

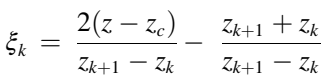

$H$ is Heaviside's function. $\delta_{i}^{k}$ and $\beta_{i}^{k}(\mathrm{i} \quad 1,2,3)$ are the continuity coefficients which depend only on normal coordinate $z$ and shear modulus of layers (i.e. $\bar{C}_{55}^{k}$ ). Some of these coefficients can be computed from the continuity requirements $U_{b 3}^{k}$ at the interface of adjacent layers. Other coefficients can be obtained from the necessity that the bending contribution of transverse shear stress in $x-z$ plane is continuous at the interface of adjacent layers and disappears at the upper and lower surfaces of the beam. For more details about coefficients $\delta_{i}^{k}$ and $\beta_{i}^{k}$, interested readers can refer to (Vidal and Polit, 2008; Beheshti-Aval et al., 2011; Lezgy-Nazargah et al., 2015; Beheshti-Aval and Lezgy-Nazargah, 2010). Similar to bending in $x$-y plane, shear correction factor is not required for bending in $x-z$ plane. The following expression gives the matrix form of Eq. (10):

$\mathbf{\Lambda}_{b 3}^{k} \quad \mathbf{O}_{b 3}^{k} \mathbf{u}_{b 3}$

where $\Lambda_{b 3}^{k} \quad U_{b 3}^{k} \quad V_{b 3}^{k} \quad W_{b 3}^{k}{ }^{T}, \mathbf{u}_{b 3} \quad w \quad \theta_{y} \quad u_{l o c}^{1}{ }^{T}$ and

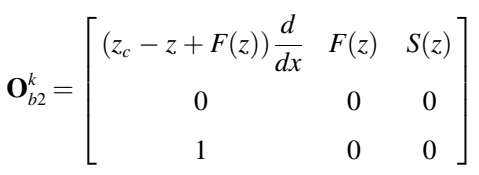

Strain components due to bending in $x-z$ plane may be expressed as the following matrix form:

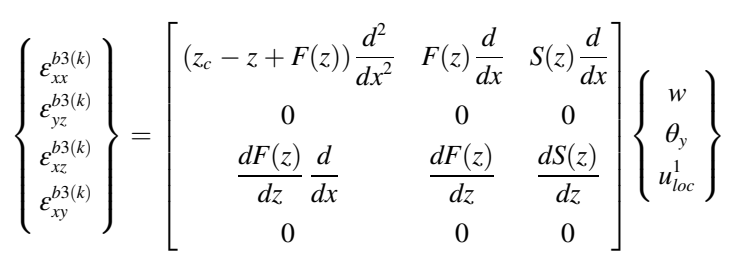

or

$\overline{\boldsymbol{\varepsilon}}_{b 3}^{k} \quad \mathbf{B}_{b 3}^{k} \mathbf{u}_{b 3}^{k}$

\subsubsection{Coupled bending and torsion-warping}

Displacement field of composite beam under coupled bending and torsional-warping loading conditions is obtained by the superimposition of displacement fields given in Eqs. (2), (7) and (10):

$$
\begin{aligned}
& U x, y, z \quad u x \quad z \quad z_{c} \frac{d w x}{d x} \quad \theta_{y} x \quad \frac{d w x}{d x} F z \quad S z u_{\text {loc }} x \\
& y \quad y_{c} \frac{d v x}{d x} \quad \theta_{z} x \quad \frac{d v x}{d x} \quad \frac{b}{\pi} \sin \frac{\pi y \quad y_{c}}{b} \quad \bar{\varphi}^{k} y, z \gamma x
\end{aligned}
$$

$W x, y, z \quad w x \quad y \quad y_{t} \theta_{x} x$

\section{Calculation of warping function}

In this section, a penalty-based 2D finite element model is derived for the calculation of unrestrained warping function $\varphi^{k} y, z$. All calculations have been carried out in the Cartesian Coordinate system $(x, y, z)$. As stated before, $y$ - and $z$-axis intersect each other at point "O", which coincides with the centroid of the composite cross-section. Indeed, the location of point "O" can be obtained based on the satisfaction of the two following equations:

$\sum_{k 1}^{N C} \int_{\Omega^{k}} C_{55}^{k} z d \Omega \quad 0$

$\sum_{k}^{N C} \int_{\Omega^{k}} C_{66}^{k} y d \Omega \quad 0$

\subsection{Weak formulation}

The following Laplace equation with Neumann boundary conditions must be satisfied by the warping function $\varphi^{k} y, z$ (Connor, 1976):

$C_{66}^{k} \frac{\partial^{2} \varphi^{k}}{\partial y^{2}} \quad C_{55}^{k} \frac{\partial^{2} \varphi^{k}}{\partial z^{2}} \quad 0 \quad$ on $\quad \Omega^{k}$

$C_{66}^{k} \frac{\partial \varphi^{k}}{\partial y} \quad z \quad \lambda_{y}^{k} \quad C_{55}^{k} \frac{\partial \varphi^{k}}{\partial z} \quad y \quad \lambda_{z}^{k} \quad 0 \quad$ in $\quad \Gamma^{k}$

where are $\Omega^{k}$ and $\Gamma^{k}$ denote the domain and boundary of kth layer, respectively. $\lambda_{y}^{k}$ and $\lambda_{z}^{k}$ are the components of unit vector normal to the boundary $\Gamma^{k}$. Multiplying Eq. (18.a) by the virtual warping function $\delta \varphi^{k}$, and integrating it over $\Omega$ gives:

$\sum_{k=1}^{N C} \int_{\Omega^{k}} \delta \varphi^{k} \quad C_{66}^{k} \frac{\partial^{2} \varphi^{k}}{\partial y^{2}} \quad C_{55}^{k} \frac{\partial^{2} \varphi^{k}}{\partial z^{2}} d \Omega \quad 0$

Based on product rule of differentiation, we have

$\frac{\partial}{\partial y} \delta \varphi^{k} \frac{\partial \varphi^{k}}{\partial y} \quad \frac{\partial}{\partial y} \delta \varphi^{k} \quad \frac{\partial \varphi^{k}}{\partial y} \quad \delta \varphi^{k} \frac{\partial^{2} \varphi^{k}}{\partial y^{2}}$

$\frac{\partial}{\partial z} \delta \varphi^{k} \frac{\partial \varphi^{k}}{\partial z} \quad \frac{\partial}{\partial z} \delta \varphi^{k} \frac{\partial \varphi^{k}}{\partial z} \quad \delta \varphi^{k} \frac{\partial^{2} \varphi^{k}}{\partial z^{2}}$

Thus, Eq. (19) can be rewritten as follow:

$$
\begin{gathered}
\sum_{k 1}^{N C} \int_{\Omega^{k}} \delta \varphi^{k} C_{66}^{k} \frac{\partial}{\partial y} \delta \varphi^{k} \frac{\partial \varphi^{k}}{\partial y} \quad C_{55}^{k} \frac{\partial}{\partial z} \delta \varphi^{k} \frac{\partial \varphi^{k}}{\partial z} \quad C_{66}^{k} \frac{\partial}{\partial y} \delta \varphi^{k} \frac{\partial \varphi^{k}}{\partial y} \\
C_{55}^{k} \frac{\partial}{\partial z} \delta \varphi^{k} \frac{\partial \varphi^{k}}{\partial z} d \Omega \quad 0
\end{gathered}
$$

By using the Gauss Divergence Theorem, the first two area integral terms of Eq. (21) can be converted to line integrals as below:

$$
\begin{array}{lll}
\int_{\Omega^{k}} \frac{\partial}{\partial y} & \delta \varphi^{k} \frac{\partial \varphi^{k}}{\partial y} d \Omega & \oint_{\Gamma^{k}} \delta \varphi^{k} \frac{\partial \varphi^{k}}{\partial y} \lambda_{y}^{k} d \Gamma \\
\int_{\Omega^{k}} \frac{\partial}{\partial z} \delta \varphi^{k} \frac{\partial \varphi^{k}}{\partial z} d \Omega & \oint_{\Gamma^{k}} \delta \varphi^{k} \frac{\partial \varphi^{k}}{\partial z} \lambda_{z}^{k} d \Gamma
\end{array}
$$

Substitution of the above two equations into Eq. (21) gives: 


$$
\begin{aligned}
& \sum_{k}^{N C} \int_{\Omega^{k}} C_{66}^{k} \frac{\partial}{\partial y} \delta \varphi^{k} \frac{\partial \varphi^{k}}{\partial y} \quad C_{55}^{k} \frac{\partial}{\partial z} \delta \varphi^{k} \frac{\partial \varphi^{k}}{\partial z} d \Omega \\
& \sum_{k}^{N C} \oint_{\Gamma^{k}} \delta \varphi^{k} \quad C_{66}^{k} \frac{\partial \varphi^{k}}{\partial y} \lambda_{y}^{k} \quad C_{55}^{k} \frac{\partial \varphi^{k}}{\partial z} \lambda_{z}^{k} \quad d \Gamma
\end{aligned}
$$

By considering the boundary condition of Eq. (18.b), Eq. (22) can be rewritten as below:

$$
\begin{aligned}
\sum_{k=1}^{N C} \int_{\Omega^{k}} C_{66}^{k} \frac{\partial}{\partial y} \delta \varphi^{k} \frac{\partial \varphi^{k}}{\partial y} \quad C_{55}^{k} \frac{\partial}{\partial z} \delta \varphi^{k} \frac{\partial \varphi^{k}}{\partial z} d \Omega & \\
& \sum_{k}^{N C} \oint_{\Gamma^{k}} \delta \varphi^{k} C_{66}^{k} \lambda_{y}^{k} z \quad C_{55}^{k} \lambda_{z}^{k} y d \Gamma
\end{aligned}
$$

or

$$
\begin{aligned}
\delta\left(\frac{1}{2} \sum_{k 1}^{N C} \int_{\Omega^{k}} C_{66}^{k} \frac{\partial \varphi^{k}}{\partial y} \frac{\partial \varphi^{k}}{\partial y} \quad C_{55}^{k} \frac{\partial \varphi^{k}}{\partial z} \frac{\partial \varphi^{k}}{\partial z} d \Omega\right. \\
\left.\sum_{k=1}^{N C} \oint_{\Gamma^{k}} \varphi^{k} C_{66}^{k} \lambda_{y}^{k} z \quad C_{55}^{k} \lambda_{z}^{k} y d \Gamma\right)
\end{aligned}
$$

Equation (23) represents the weak formulation of Eq. (18). At the interface between layers, the displacement component $U_{t}^{k}$, in-plane shear strain $\varepsilon_{x y}^{t k}$, and transverse shear stress $\sigma_{x z}^{t k}$ must be continuous along the interface $z_{k}$. The foresaid conditions require satisfying the following relations:

$\varphi^{k 1} y, z \quad z_{k 1} \quad \varphi^{k} y, z \quad z_{k 1}$

$\varphi_{y}^{k}{ }^{1} y, z \quad z_{k 1} \quad \varphi_{y}^{k} y, z \quad z_{k 1}$

$C_{55}^{k} \frac{\partial \varphi^{k 1} y, z \quad z_{k 1}}{\partial z} \quad C_{55}^{k} \frac{\partial \varphi^{k} y, z \quad z_{k 1}}{\partial z} \quad C_{55}^{k} \quad C_{55}^{k}{ }^{1} y$

where $k \quad 1,2,3, \ldots, N C \quad 1$. The above constraints will be tackled at the weak formulation by using the penalty approach. Note that Eq. (24. c) is obtained based on Saint-Venant assumption, i.e. $\gamma \quad d \theta_{x} / d x$. Thus, interface continuity conditions for transverse shear stresses due to warping and torsion may not completely be fulfilled at the interfaces. In the numerical evaluations of the present formulation, it will be verified that this simplifying assumption leads to results with enough accuracy.

\subsection{FE formulation}

As depicted in Fig. 2, the cross-section of composite beam is discretized into arbitrary sub-domains $\Omega_{e}^{k}$ whose boundary is $\Gamma_{e}^{k}$. It is assumed that each arbitrary sub-domain $\Omega_{e}^{k}$ represents a 4-node rectangular element. For the approximation of warping function on each element domain, the full compatible Hermite's shape functions which ensures $C^{1}$-type continuity of warping function along y and $\mathrm{z}$ directions, are employed:

$\varphi^{k} \quad \mathbf{N} \widehat{\boldsymbol{\Phi}}_{e}^{k}$

Interpolation matrix $\boldsymbol{N}$ and warping nodal variable vector $\widehat{\boldsymbol{\Phi}}_{e}^{k}$ are defined as follows:

$\widehat{\boldsymbol{\Phi}}_{e}^{k} \quad\left[\begin{array}{cccc}\widehat{\boldsymbol{\Phi}}_{g_{1}}^{k} & \widehat{\boldsymbol{\Phi}}_{g_{2}}^{k} & \widehat{\boldsymbol{\Phi}}_{g_{3}}^{k} & \widehat{\boldsymbol{\Phi}}_{g_{4}}^{k}\end{array}\right]^{T}$

$\begin{array}{lllll}\mathbf{N} & \mathbf{N}_{11} & \mathbf{N}_{21} & \mathbf{N}_{12} & \mathbf{N}_{22}\end{array}$

The sub-matrices appeared in the above Eqs. (26) and (27) are given by:

$\mathbf{N}_{f q} \quad H_{0 f} \xi H_{0 q} \eta \quad H_{1 f} \xi H_{0 q} \eta \quad H_{0 f} \xi H_{1 q} \eta \quad H_{1 f} \xi H_{1 q} \eta \quad f, q \quad 1,2$

$\widehat{\boldsymbol{\Phi}}_{g_{1}}^{k}\left[\begin{array}{llll}\widehat{\varphi}_{g_{1}}^{k} & \widehat{\varphi}_{g_{1}, y}^{k} & \widehat{\varphi}_{g_{1}, z}^{k} & \widehat{\varphi}_{g_{1}, y z}^{k}\end{array}\right]$

$\widehat{\boldsymbol{\Phi}}_{g_{2}}^{k} \quad\left[\begin{array}{llll}\widehat{\varphi}_{g_{2}}^{k} & \widehat{\varphi}_{g_{2}, y}^{k} & \widehat{\varphi}_{g_{2}, z}^{k} & \widehat{\varphi}_{g_{2}, y z}^{k}\end{array}\right]$

$\widehat{\boldsymbol{\Phi}}_{g_{3}}^{k} \quad\left[\begin{array}{llll}\widehat{\varphi}_{g_{3}}^{k} & \widehat{\varphi}_{g_{3}, y}^{k} & \widehat{\varphi}_{g_{3}, z}^{k} & \widehat{\varphi}_{g_{3}, y z}^{k}\end{array}\right]$

$\widehat{\boldsymbol{\Phi}}_{g_{4}}^{k}\left[\begin{array}{llll}\widehat{\varphi}_{g_{4}}^{k} & \widehat{\varphi}_{g_{4}, y}^{k} & \widehat{\varphi}_{g_{4, z}}^{k} & \widehat{\varphi}_{g_{4}, y z}^{k}\end{array}\right]$

where $\xi$ and $\eta$ are the local coordinates of element and are chosen to be along $y$ and $z$ directions, respectively. $H_{\alpha \kappa} \chi \quad \alpha \quad 0,1 ; \kappa \quad 1,2 ; \chi \quad \xi, \eta$ are the classical Hermite's shape functions:

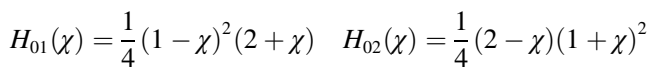

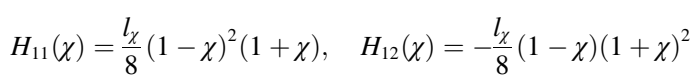

$l_{\chi}$ is the length of the element along $\chi$ direction. It is worth to be noted that the vector $\widehat{\boldsymbol{\Phi}}_{e}^{k}$ contains the nodal values of the warping function and its derivatives. Consideration of $\widehat{\varphi}_{i, y}^{k}$ and $\widehat{\varphi}_{j . y}^{k}$ as independent degrees of freedom guarantees the continuity of transverse shear stress $\sigma_{x y}^{t k}$ and $\sigma_{x z}^{t k}$ between adjacent elements of the kth layer. Substituting Eq. (25) into (23) yields:

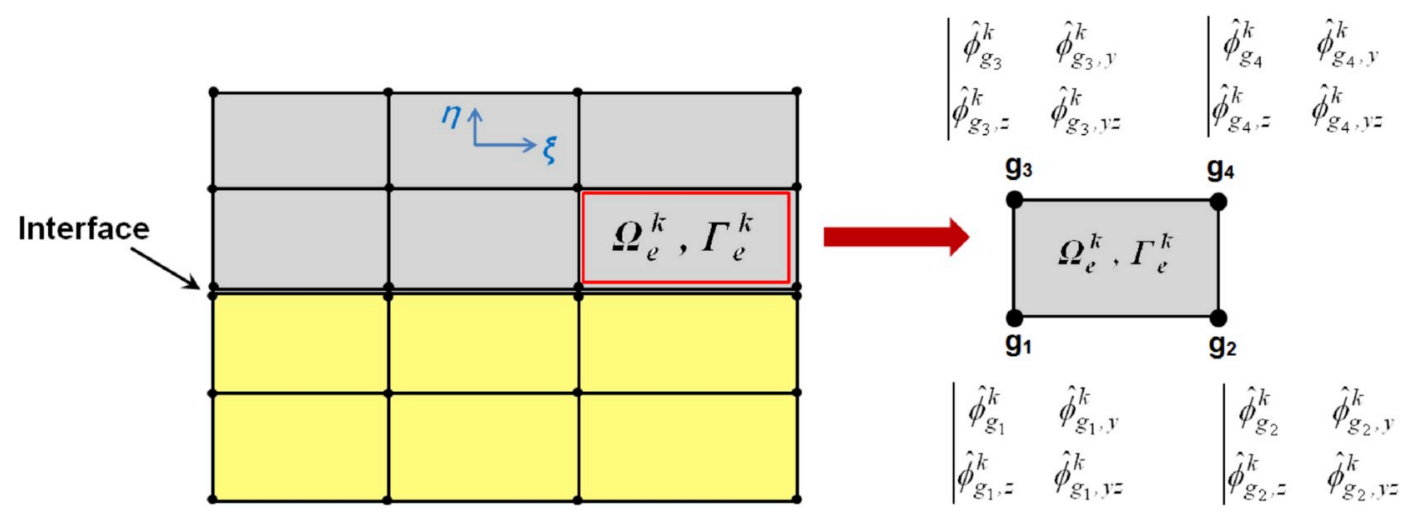

Fig. 2. Discretization of the cross-section by using 4-node Hermitian elements. 


$$
\begin{aligned}
&\left.\delta \frac{1}{2} \sum_{k 1}^{N C} \sum^{m_{e}^{k}} \int_{\Omega_{e}^{k}} C_{66}^{k} \widehat{\boldsymbol{\Phi}}_{e}^{k T} \frac{\partial \mathbf{N}^{T}}{\partial y} \frac{\partial \mathbf{N}}{\partial y} \widehat{\boldsymbol{\Phi}}_{e}^{k} \quad C_{55}^{k} \widehat{\boldsymbol{\Phi}}_{e}^{k T} \frac{\partial \mathbf{N}^{T}}{\partial z} \frac{\partial \mathbf{N}}{\partial z} \widehat{\boldsymbol{\Phi}}_{e}^{k} d \Omega\right) \\
&\left.\left.\left.\sum_{k 1}^{N C} \sum^{m_{e}^{k}} \int_{\Gamma_{e}^{k}} \widehat{\boldsymbol{\Phi}}_{e}^{k T} \mathbf{N}^{T} \quad C_{66}^{k} \lambda_{y}^{k} z \quad C_{55}^{k} \lambda_{z}^{k} y\right) d \Gamma\right)\right) 0
\end{aligned}
$$

where $m_{e}^{k}$ denotes the number of cross-sectional elements of kth layer. In the above discrete weak formulation, the constraint conditions (24.a)(24.c) are not considered. For satisfying these constraints by using the penalty finite element approach, Eq. (38) must be modified as below:

$$
\begin{aligned}
& \left.\delta \frac{1}{2} \sum_{k 1}^{N C} \sum^{m_{e}^{k}} \int_{\Omega_{e}^{k}} C_{66}^{k} \widehat{\boldsymbol{\Phi}}_{e}^{k T} \frac{\partial \mathbf{N}^{T}}{\partial y} \frac{\partial \mathbf{N}}{\partial y} \widehat{\boldsymbol{\Phi}}_{e}^{k} \quad C_{55}^{k} \widehat{\boldsymbol{\Phi}}_{e}^{k T} \frac{\partial \mathbf{N}^{T}}{\partial z} \frac{\partial \mathbf{N}}{\partial z} \widehat{\boldsymbol{\Phi}}_{e}^{k} d \Omega\right) \\
& \left.\sum_{k=1}^{N C} \sum^{m_{e}^{k}} \int_{\Gamma_{e}^{k}} \widehat{\boldsymbol{\Phi}}_{e}^{k T} \mathbf{N}^{T} \quad C_{66}^{k} \lambda_{y}^{k} z \quad C_{55}^{k} \lambda_{z}^{k} y d \Gamma\right) \\
& \left.\left.\frac{1}{2} p_{\varphi} \sum_{k=1}^{N C} \sum_{i, j 1}^{1} \widehat{\varphi}_{i}^{n_{z k} 1} \hat{\varphi}^{k} 1 \widehat{\varphi}_{j}^{k}{ }^{2}\right) \quad \frac{1}{2} p_{\varphi_{y}} \sum_{k=1}^{N C} \sum_{i, j 1}^{1} \widehat{\varphi}_{i, y}^{n_{z k} 1} \quad \widehat{\varphi}_{j, y}^{k}{ }^{2}\right) \\
& \left.\left.\frac{1}{2} p_{\varphi_{, z}} \sum_{k=1}^{N C 1} \sum_{i, j 1}^{n_{z k 1}} C_{55}^{k 1} \widehat{\varphi}_{i, z}^{k 1} \quad C_{55}^{k} \widehat{\varphi}_{j, z}^{k} \quad C_{55}^{k} \quad C_{55}^{k}{ }^{1} y_{i}{ }^{2}\right)\right) 0
\end{aligned}
$$

where $p_{\varphi}, p_{\varphi_{y y}}$ and $p_{\varphi_{, z}}$ are the penalty parameters and $n_{z_{k} 1}$ denotes the number of adjacent nodes along the interface $z \quad z_{k}$. Eq. (39) leads to a set of simultaneous equations to be solved for the nodal values of the trial solution $\varphi^{k} y, z$. The matrix form of this set of equations is as follow:

$$
\begin{array}{lllllll}
\mathbf{K} & \mathbf{K}_{\varphi} & \mathbf{K}_{\varphi_{y,}} & \mathbf{K}_{\varphi_{, z}} & \widehat{\boldsymbol{\Phi}} & \mathbf{Q} & \mathbf{Q}_{\varphi_{, z}}
\end{array}
$$

where

$$
\mathbf{K} \quad \sum^{m_{e}} \mathbf{K}^{e}, \quad \widehat{\boldsymbol{\Phi}} \quad \sum^{m_{e}} \widehat{\boldsymbol{\Phi}}_{e}^{k}, \quad \mathbf{Q} \quad \sum^{m_{e}} \mathbf{Q}^{e}
$$

and $m_{e}$ denotes the total number of cross-sectional elements. The elementary matrices $\mathbf{K}^{e}$ and $\mathbf{Q}^{e}$ are defined as follows:

$$
\begin{aligned}
& \mathbf{K}^{e} \quad \int_{\Omega_{e}^{k}} \frac{\partial \mathbf{N}^{T}}{\partial y} \frac{\partial \mathbf{N}}{\partial y} \frac{\partial \mathbf{N}^{T}}{\partial z} \frac{\partial \mathbf{N}}{\partial z} d y d z \\
& \mathbf{Q}^{e} \quad \oint_{\Gamma_{e}^{k}} \mathbf{N}^{T} \quad \lambda_{y}^{k} z \quad \lambda_{z}^{k} y \quad d \Gamma
\end{aligned}
$$

$\mathbf{K}_{\varphi}, \mathbf{K}_{\varphi_{y}}$ and $\mathbf{K}_{\varphi_{, z}}$ are $n \quad n$ matrices, which $n$ denotes the total number of nodal variables. $\mathbf{Q}_{\varphi_{z}}$ is a vector with size $n \quad 1$. Details about the calculation of arrays of matrices $\mathbf{K}_{\varphi}, \mathbf{K}_{\varphi_{y}}, \mathbf{K}_{\varphi_{z}}$ and $\mathbf{Q}_{\varphi_{z}}$ are given in Appendix A.

Thus, one can calculate the nodal values of the warping function from Eq. (40). The torsional $(J)$ and warping $C_{w}$ constants, which are cross-sectional properties, can be also obtained from the following relations (Connor, 1976):

$$
\begin{aligned}
& J \quad \int_{\Omega} y^{2} \quad z^{2} \quad y \frac{\partial \varphi}{\partial z} \quad z \frac{\partial \varphi}{\partial y} d \Omega \\
& C_{w} \quad \int_{\Omega} \varphi^{2} y, z d \Omega
\end{aligned}
$$

Remark. The 2D finite element introduced in this section is not restricted to rectangular laminated cross-sections. It can be easily used for the calculation of torsional-warping properties of composite beams with arbitrary cross-sections by modifying the constraint conditions (24. a)-(24.c).

\section{FE formulation for the composite beam}

\subsection{Interpolation of displacement field variables}

A 1D FE formulation is developed here for the prediction of structural responses of composite beams under combined loads. To this aim, the unknown variables of displacement field must be approximated properly. In addition to warping function $\varphi^{k} y, z$, the present beam model has eight unknown field variables $u x, v x, w x, u_{l o c} x, \theta_{x} x$, $\theta_{y} x, \theta_{z} x$ and $\gamma x$. It is assumed that the composite beam is longitudinally divided into $n_{e}$ multifiber elements. A multifiber element with length $l_{e}$ is shown in Fig. 3. Every longitudinal multifiber element is made of $m_{e}$ fibers. It should be noted that the number and the geometrical properties of the fibers must be in accordance with 2D mesh used for solving the warping function $\varphi^{k} y, z$. As shown in Fig. 3, every fiber of beam can be mathematically replaced with a $1 \mathrm{D}$ three-node fiber element.

Based on assumed kinematic (i.e. Eq. (16)), the bending deflections $v x, w x$ should be $\mathrm{C}^{1}$-continuous over domain of a fiber. Thus, the variables $v x, w x$ at each fiber are interpolated by classic Hermite cubic functions. $\mathrm{C}^{0}$-continuity is enough for the interpolation of other field variables. They can be approximated via linear Lagrange's shape functions. However, quadratic Lagrange's shape functions are employed for the interpolation of bending variables $\theta_{y} x, \theta_{z} x$ and $u_{l o c} x$. Due to the field consistency principle (Ganapathi et al., 1999; Vidal and Polit, 2008; Beheshti-Aval et al., 2011; Lezgy-Nazargah et al., 2015), this interpolation choice prevents from the appearance of transverse shear stress locking phenomena. Finally, $u x, \theta_{x} x$, and $\gamma x$ are interpolated by using linear Lagrangian shape functions. Therefore, unknown field variables $u x, v x, w x, u_{l o c} x, \theta_{x} x, \theta_{y} x, \theta_{z} x$ and $\gamma x$ can be written with respect to their nodal variables as below:
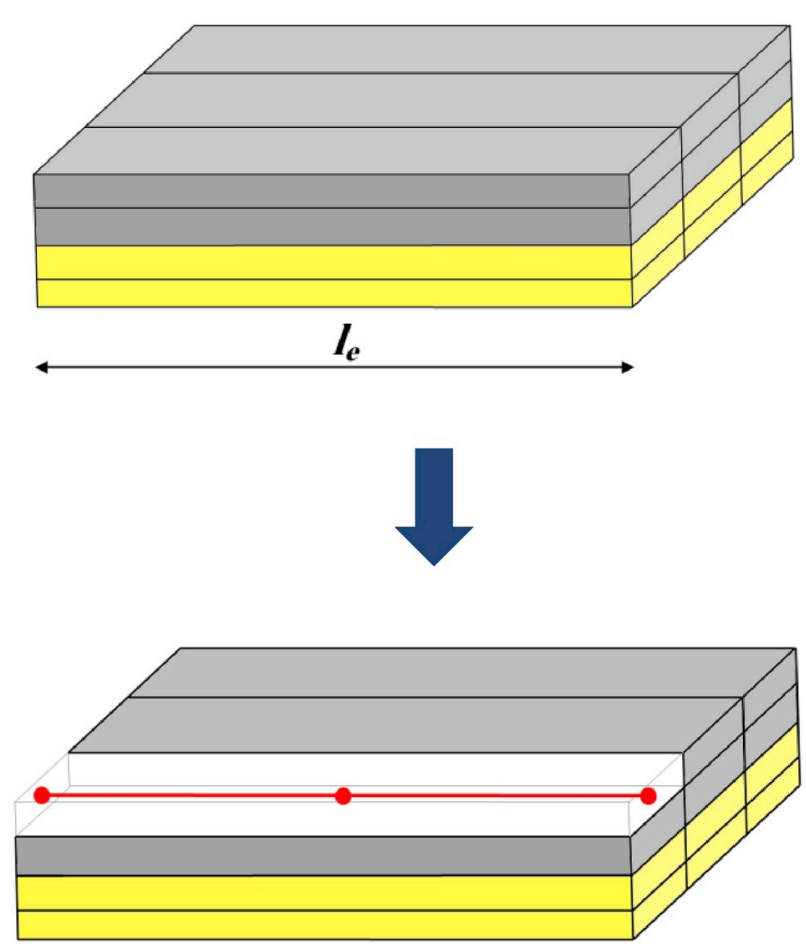

Fig. 3. Description of the composite beam element and constitutive fibers. 
$u \quad \mathbf{N}_{\mathbf{L L}} \widehat{\mathbf{u}}_{u}^{\mathbf{e}}$

$v \quad \mathbf{N}_{\mathbf{H}} \widehat{\mathbf{u}}_{\mathbf{y}}^{\mathbf{e}}$

$w \quad \mathbf{N}_{\mathbf{H}} \widehat{\mathbf{u}}_{\mathbf{w}}^{\mathbf{e}}$

$\theta_{y} \quad \mathbf{N}_{\mathbf{Q L}} \widehat{\mathbf{u}}_{\theta_{y}}^{\mathbf{e}}$

$\theta_{z} \quad \mathbf{N}_{\mathbf{Q L}} \widehat{\mathbf{u}}_{\boldsymbol{\theta}_{\boldsymbol{z}}}^{\mathbf{e}}$

$u_{l o c} \quad \mathbf{N}_{\mathbf{Q L}} \widehat{\mathbf{u}}_{\mathbf{u}_{\text {loc }}}^{\mathrm{e}}$

$\theta_{x} \quad \mathbf{N}_{\mathbf{L L}} \widehat{\mathbf{u}}_{\boldsymbol{\theta}_{\mathbf{x}}}^{\mathbf{e}}$

$\gamma \quad \mathbf{N}_{\mathbf{L L}} \widehat{\mathbf{u}}_{\gamma}^{\mathbf{e}}$

$\widehat{\mathbf{u}}_{\mathbf{u}}^{e}, \widehat{\mathbf{u}}_{\mathbf{v}}^{\mathbf{e}}, \widehat{\mathbf{u}}_{\mathbf{w}}^{\mathbf{e}}, \widehat{\mathbf{u}}_{\boldsymbol{\theta}_{\mathbf{y}}}^{\mathbf{e}}, \widehat{\mathbf{u}}_{\boldsymbol{\theta}_{z}}^{\mathbf{e}}, \widehat{\mathbf{u}}_{\boldsymbol{\theta}_{\mathbf{x}}}^{\mathbf{e}}, \widehat{\mathbf{u}}_{\mathbf{u}_{\mathrm{loc}}}^{\mathbf{e}}$ and $\widehat{\mathbf{u}}_{\gamma}^{\mathrm{e}}$ are the vectors of degrees of freedom of the fiber elements while $\mathbf{N}_{\mathrm{LL}}, \mathbf{N}_{\mathbf{H}}$ and $\mathbf{N}_{\mathrm{QL}}$ are interpolating matrices. Details of the mentioned vectors and matrices are given in Appendix B.

The considered 3-node fiber element and its assumed local coordinate system are shown in Fig. 4. Note that all constitutive fibers of a beam element have the same degrees of freedom. Only nodal values related to warping function $\varphi^{k} y, z$ are different at each fiber element.

By substituting Eq. (43) into Eqs. (5), (8) and (14), the displacement components of composite beam can be rewritten in the following matrix forms:

$\Lambda_{t}^{k} \quad \mathbf{O}_{t}^{k} \mathbf{N}_{t} \widehat{\mathbf{u}}_{t}^{e}$

$\mathbf{\Lambda}_{b 2}^{k} \quad \mathbf{O}_{b 2}^{k} \mathbf{N}_{b 2} \widehat{\mathbf{u}}_{b 2}^{e}$

$\Lambda_{b 3}^{k} \quad \mathbf{O}_{b 3}^{k} \mathbf{N}_{b 3} \widehat{\mathbf{u}}_{b 3}^{e}$

$\begin{array}{lllllllllll}\text { where } & \widehat{\mathbf{u}}_{t}^{e} & \widehat{\mathbf{u}}_{\theta_{x}}^{e} & \widehat{\mathbf{u}}_{\gamma}^{e} & T, & \widehat{\mathbf{u}}_{b 2}^{e} & \widehat{\mathbf{u}}_{u}^{e} & \widehat{\mathbf{u}}_{v}^{e} & \widehat{\mathbf{u}}_{\theta_{z}}^{e} & T, & \widehat{\mathbf{u}}_{b 3}^{e}\end{array}$ $\begin{array}{llll}\widehat{\mathbf{u}}_{w}^{e} & \widehat{\mathbf{u}}_{\theta_{y}}^{e} & \widehat{\mathbf{u}}_{u_{l o c}}^{e} & { }^{T}\end{array}$

$\mathbf{N}_{t} \quad\left[\begin{array}{cc}\mathbf{N}_{L L}^{T} & \mathbf{0} \\ \mathbf{0} & \mathbf{N}_{L L}^{T}\end{array}\right], \mathbf{N}_{b 2}\left[\begin{array}{ccc}\mathbf{N}_{L L}^{T} & \mathbf{0} & \mathbf{0} \\ \mathbf{0} & \mathbf{N}_{H}^{T} & \mathbf{0} \\ \mathbf{0} & \mathbf{0} & \mathbf{N}_{Q L}^{T}\end{array}\right], \mathbf{N}_{b 3}\left[\begin{array}{ccc}\mathbf{N}_{H}^{T} & \mathbf{0} & \mathbf{0} \\ \mathbf{0} & \mathbf{N}_{Q L}^{T} & \mathbf{0} \\ \mathbf{0} & \mathbf{0} & \mathbf{N}_{Q L}^{T}\end{array}\right]$

Similarly, strain components of composite beam (Eq. (6), (9) and (15)) can be expressed as:

$\overline{\boldsymbol{\varepsilon}}_{t}^{k} \quad \mathbf{B}_{t}^{k} \mathbf{O}_{t}^{k} \mathbf{N}_{t} \widehat{\mathbf{u}}_{t} \quad \mathbf{A}_{t}^{k} \widehat{\mathbf{u}}_{t}^{e}$

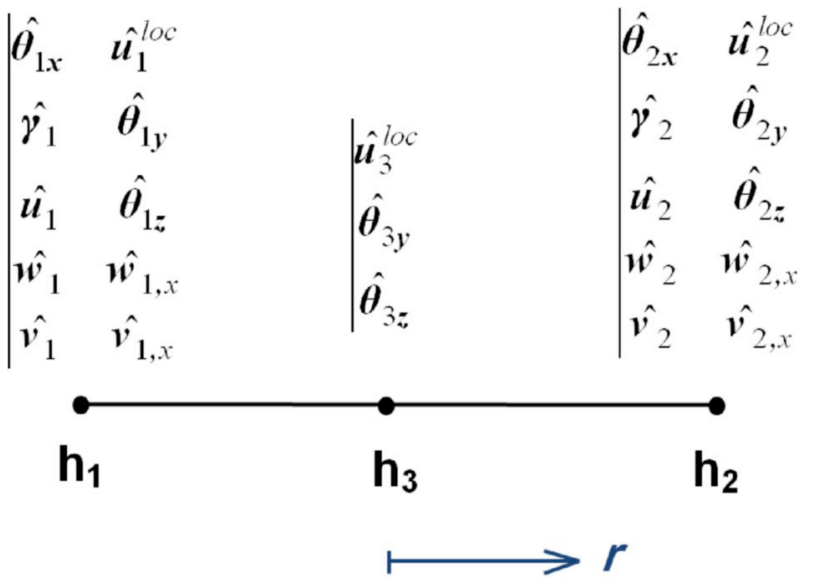

Fig. 4. Three-node fiber element: local coordinate system and nodal degrees of freedom. $\overline{\boldsymbol{\varepsilon}}_{b 2}^{k} \quad \mathbf{B}_{b 2}^{k} \mathbf{O}_{b 2}^{k} \mathbf{N}_{b 2} \widehat{\mathbf{u}}_{b 2} \quad \mathbf{A}_{b 2}^{k} \widehat{\mathbf{u}}_{b 2}$

$\overline{\mathbf{\varepsilon}}_{b 3}^{k} \quad \mathbf{B}_{b 3}^{k} \mathbf{O}_{b 3}^{k} \mathbf{N}_{b 3} \widehat{\mathbf{u}}_{b 3} \quad \mathbf{A}_{b 3}^{k} \widehat{\mathbf{u}}_{b 3}$

\subsection{Weak formulation of the boundary value problem}

The total potential energy of the considered composite beam can be represented as below:

$\Pi \quad \sum^{n_{e}} \int_{0}^{l_{e}} \sum^{m_{e}} \int_{\Omega_{e}} \overline{\boldsymbol{\varepsilon}}_{t}^{k T} \boldsymbol{\sigma}_{t}^{k} \quad \overline{\boldsymbol{\varepsilon}}_{b 2}^{k T} \boldsymbol{\sigma}_{b 2}^{k} \quad \overline{\boldsymbol{\varepsilon}}_{b 3}^{k T} \boldsymbol{\sigma}_{b 3}^{k} d y d z d x$

$\sum^{n_{e}} \int_{0}^{l_{e}} \sum^{m_{e}} \int_{\Omega_{e}} \boldsymbol{\Lambda}_{t}^{k T} \quad \boldsymbol{\Lambda}_{b 2}^{k T} \quad \boldsymbol{\Lambda}_{b 3}^{k T} \overline{\mathbf{p}} d y d z d x$

$\left.\sum^{n_{e}} \int_{0}^{l_{e}} \sum_{k 1}^{N C} \boldsymbol{\Lambda}_{t}^{k T} \quad \boldsymbol{\Lambda}_{b 2}^{k T} \quad \boldsymbol{\Lambda}_{b 3}^{k T} \overline{\mathbf{P}}_{s}\right) d x$

where $\overline{\mathbf{P}}_{V}$ and $\overline{\mathbf{P}}_{s}$ vector of body and surface loads, respectively. Substituting Eqs. (1) and (44)-(45) into the above equation gives the total potential energy with respect to the nodal variables of composite beam. Based on the principle of minimum potential energy, the nodal variables of beam can be obtained by solving the following $n \quad n$ linear system of equations:

$\mathbf{K}_{\text {beam }} \widehat{\mathbf{u}} \mathbf{F}$

where $\mathrm{n}$ denotes the total number of nodal variables of composite beam. $\mathbf{K}, \widehat{\mathbf{u}}$ and $\mathbf{F}$ are total stiffness matrix, nodal variables vector and total force vector, respectively. The aforementioned matrices are obtained after calculation and assembling the corresponding elementary matrices as below:

$$
\begin{gathered}
\mathbf{K}_{\text {beam }} \sum^{m_{e} n_{e}}\left[\begin{array}{ccc}
\mathbf{K}_{t}^{e} & \mathbf{0} & \mathbf{0} \\
\mathbf{0} & \mathbf{K}_{b 2}^{e} & \mathbf{0} \\
\mathbf{0} & \mathbf{0} & \mathbf{K}_{b 3}^{e}
\end{array}\right], \quad \widehat{\mathbf{u}} \sum^{m_{e}} n_{e}\left\{\begin{array}{c}
\widehat{\mathbf{u}}_{t}^{e} \\
\widehat{\mathbf{u}}_{b 2}^{e} \\
\widehat{\mathbf{u}}_{b 3}^{e}
\end{array}\right\}, \quad \mathbf{F} \quad \sum^{m_{e}} n_{e}\left\{\begin{array}{c}
\mathbf{f}_{t}^{\mathbf{e}} \\
\mathbf{f}_{b 2}^{\mathbf{e}} \\
\mathbf{f}_{b 3}^{\mathbf{e}}
\end{array}\right\} \\
\sum^{N C n_{e}}\left\{\begin{array}{c}
\mathbf{p}_{t}^{\mathbf{e}} \\
\mathbf{p}_{b 2}^{\mathbf{e}} \\
\mathbf{p}_{b 3}^{\mathbf{e}}
\end{array}\right\}
\end{gathered}
$$

where

$$
\begin{array}{ll}
\mathbf{K}_{l}^{e} & \int_{0}^{l_{e}} \int_{\Omega_{e}} \mathbf{A}_{l}^{k T} \overline{\mathbf{C}}^{k} \mathbf{A}_{\iota}^{k} d y d z d x, \quad l \quad t, b 2, b 3 \\
\mathbf{f}_{l}^{e} & \int_{0}^{l_{e}} \int_{\Omega_{e}} \mathbf{N}_{l}^{T} \mathbf{O}_{l}^{k T} \overline{\mathbf{P}}_{\mathbf{V}} d y d z d x, \quad l \quad t, b 2, b 3 \\
\mathbf{p}_{t}^{\mathbf{e}} & \int_{0}^{l_{e}} \mathbf{N}_{l}^{T} \mathbf{O}_{l}^{k T} \overline{\mathbf{P}}_{s} d x, \quad l \quad t, b 2, b 3
\end{array}
$$

Remark. on the present finite element model. The multifiber finite element formulation introduced in the previous sections, indeed, decouples the problem of 3D analysis of laminated composite and sandwich beams under combined loads into 2D cross-sectional analysis and 1D beam analysis. This concept is shown schematically in Fig. 5.

\section{Numerical results}

The aim of this section is to evaluate the accuracy of the presented penalty-based finite element model for the bending and torsionalwarping analysis of composite beams. Composite beams with various 


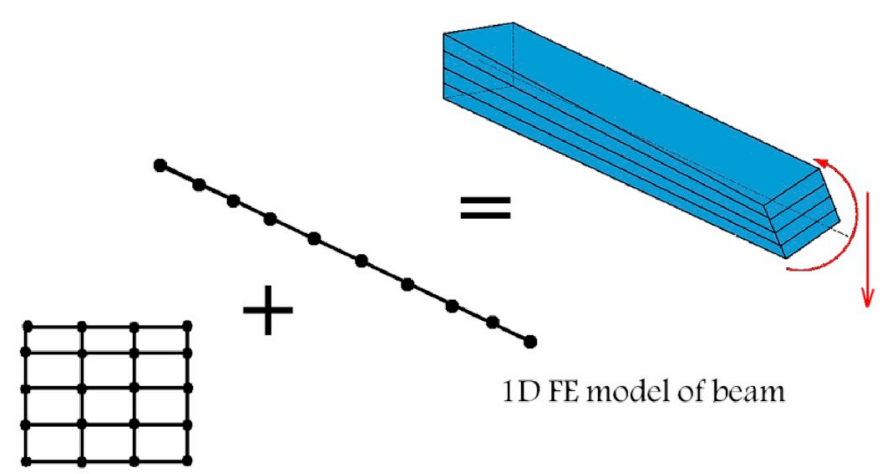

2D FE model of cross-section

Fig. 5. Modeling of composite beams by using the present formulation: schematic representation.

cross-sections are analyzed by using the present formulation. The results of 3D finite element analysis as well as the previous published reports are employed for the comparison purposes.

\subsection{Cross-sectional analysis of composite sections with different configurations}

In this example, torsional rigidity and coordinates of twist center of some composite cross-sections with different configurations are determined by using the penalty-based finite element model described in Section 3. The geometrical characteristics of considered composite cross-sections as well as considered Coordinate system $(\mathrm{Y}, \mathrm{Z})$ are shown in Fig. 6. In this figures, Y- and Z-axes intersect each other at centroid of homogenous cross-section.

The convergence of the present penalty-based finite element model to evaluate the torsional properties of composite cross-sections is studied in Table 1 . In this table, $\widetilde{G J}$ denotes the torsional rigidity of composite cross-section while $G J_{1}$ is the torsional rigidity of the corresponding homogenous cross-section made of material 1. Mechanical properties assumed for the material 1 and 2 are given in Table 1 . It is seen that the proposed finite element model has a fast convergence and few elements are needed to predict the torsional constant as well as the position of twist center.

In Table 2, the accuracy of the present results are evaluated through comparison with other available results. 2D finite element results of Saygun et al. (2007) and 3D finite element results of El Fatmi and Zenzri (El Fatmi and Zenzri, 2004) are chosen for comparison purposes. One can see that the present results are in good agreement with those of previous works. Saygun et al. (2007) carried out their numerical implementations based on Saint-Venant's stress function by using 4-noded 2D elements. The results of El Fatmi and Zenzri (El Fatmi and Zenzri, 2004) is based on an exact elastic beam theory. They used 3D 20-node brick elements for the computations of operators involved in their beam theory. However, the present results are obtained by using lower number of elements and degrees of freedom.

\subsection{Cross-sectional analysis of a three-part composite section}

Torsion and warping cross-sectional properties of a composite beam are calculated in this section. The considered composite beam is a cantilevered one with length $\mathrm{L} \quad 6 \mathrm{~m}$. The geometrical characteristic of the beam cross-section as well as the considered Coordinate system are depicted in Fig. 7. The origin of the Coordinate system $(y, z)$ is coincided on the centroid of composite cross-section. As shown in Fig. 7, the crosssection of the beam is made of three rectangular parts with different materials. Mechanical properties of materials 1, 2 and 3 are given in Table 3.

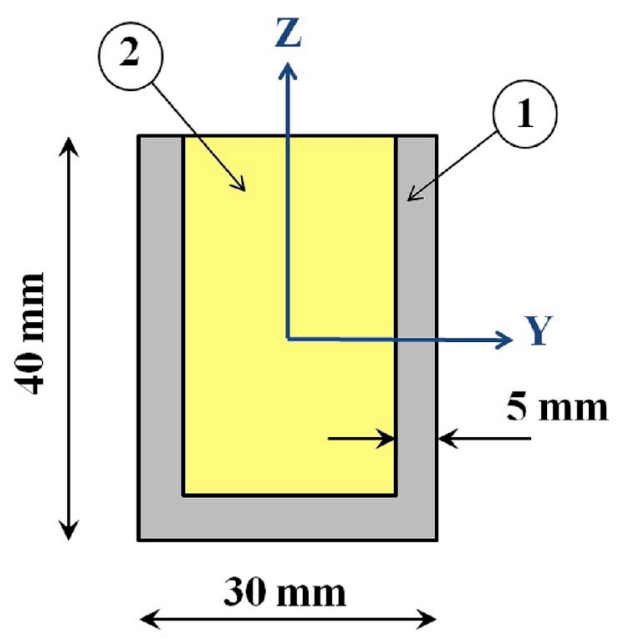

(a)

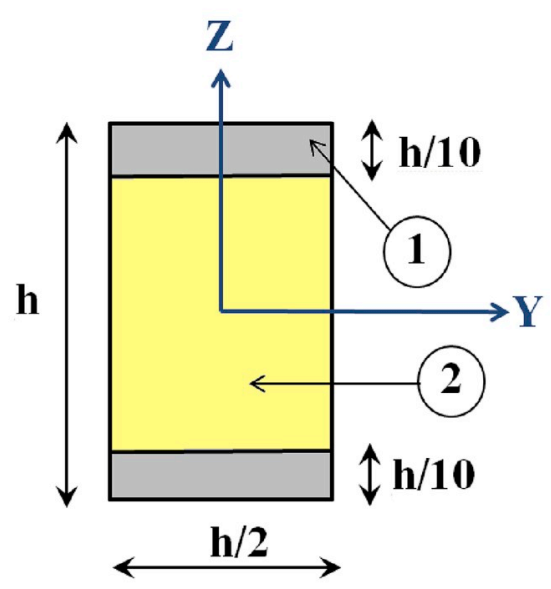

(b)

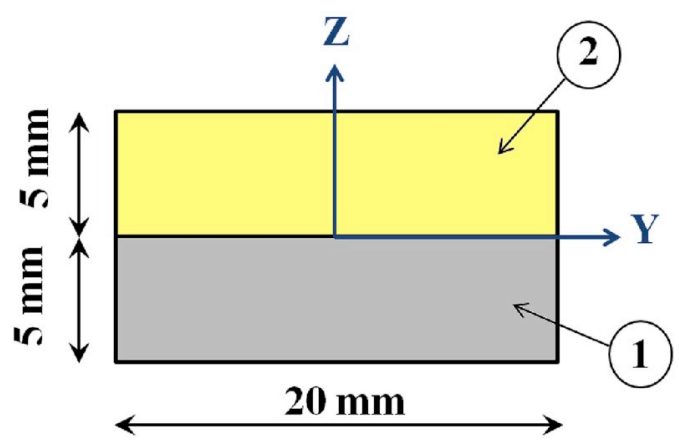

(c)

Fig. 6. Geometrical and mechanical characteristics of composite cross-section with different configurations: (a) Case 1, (b) Case 2, (c) Case 3. 
Table 1

Results of mesh convergence study to evaluate the torsional properties of composite cross-sections.

\begin{tabular}{|c|c|c|c|c|c|}
\hline \multirow{2}{*}{$\begin{array}{l}\text { Case no. } \\
1\end{array}$} & \multicolumn{2}{|c|}{ Mechanical properties } & \multirow{2}{*}{$\begin{array}{l}\text { No. elements } \\
48\end{array}$} & \multirow{2}{*}{$\begin{array}{c}\widetilde{G J} / G J_{1} \\
0.5286\end{array}$} & \multirow{2}{*}{$\begin{array}{l}y_{t}, z_{t} \\
(0.00,-4.84)\end{array}$} \\
\hline & $E_{1}$ & $72.95 \mathrm{GPa}$ & & & \\
\hline & $E_{2}$ & $10.67 \mathrm{GPa}$ & 192 & 0.5264 & $(0.00,-4.85)$ \\
\hline & $v_{1}$ & $\begin{array}{ll}v_{2} & 0.33\end{array}$ & 384 & 0.5264 & $(0.00,-4.85)$ \\
\hline & & & 768 & 0.5264 & $(0.00,-4.85)$ \\
\hline \multirow[t]{5}{*}{2} & \multicolumn{2}{|c|}{$E_{1} / E_{2} \quad 50$} & 3 & 0.0307 & $(0.00,0.00)$ \\
\hline & $v_{1}$ & 0 & 12 & 0.0306 & $(0.00,0.00)$ \\
\hline & \multicolumn{2}{|c|}{$v_{2} \quad 0.45$} & 48 & 0.0305 & $(0.00,0.00)$ \\
\hline & & & 96 & 0.0305 & $(0.00,0.00)$ \\
\hline & & & 192 & 0.0305 & $(0.00,0.00)$ \\
\hline \multirow[t]{6}{*}{3} & $E_{1}$ & $1 \mathrm{GPa}$ & 2 & 0.8262 & $(0.00,-0.721)$ \\
\hline & $E_{2}$ & $1 \mathrm{GPa}$ & 8 & 0.8261 & $(0.00,-0.722)$ \\
\hline & $v_{1}$ & 0 & 50 & 0.8261 & $(0.00,-0.722)$ \\
\hline & \multirow[t]{3}{*}{$v_{2}$} & \multirow[t]{3}{*}{0.45} & 100 & 0.8261 & $(0.00,-0.722)$ \\
\hline & & & 200 & 0.8261 & $(0.00,-0.722)$ \\
\hline & & & 800 & 0.8261 & $(0.00,-0.722)$ \\
\hline
\end{tabular}

Table 2

Comparison of torsional properties of composite cross-sections obtained by different methodes

\begin{tabular}{|c|c|c|c|c|}
\hline & & $\begin{array}{l}\text { El Fatmi and Zenzri (El } \\
\text { Fatmi and Zenzri, 2004) }\end{array}$ & $\begin{array}{l}\text { Saygun et al. ( } \\
\text { Saygun et al., } \\
\text { 2007) }\end{array}$ & Present \\
\hline \multirow{3}{*}{$\begin{array}{c}\text { Case } \\
1\end{array}$} & $\widetilde{G J} / G J_{1}$ & 0.5249 & 0.5250 & 0.5264 \\
\hline & $y_{t}, z_{t}$ & $(0.0,-4.90)$ & - & $\begin{array}{l}(0.00,- \\
4.85)\end{array}$ \\
\hline & $\begin{array}{l}\text { No. } \\
\text { elements }\end{array}$ & 200 & 3072 & 192 \\
\hline \multirow{3}{*}{$\begin{array}{c}\text { Case } \\
2\end{array}$} & $\widetilde{G J} / G J_{1}$ & 0.0307 & 0.0391 & 0.0305 \\
\hline & $y_{t}, z_{t}$ & $(0.00,0.00)$ & - & $(0.00,0.00)$ \\
\hline & $\begin{array}{l}\text { No. } \\
\text { elements }\end{array}$ & 200 & 1250 & 48 \\
\hline \multirow{3}{*}{$\begin{array}{c}\text { Case } \\
3\end{array}$} & $\widetilde{G J} / G J_{1}$ & 0.8251 & 0.8250 & 0.8261 \\
\hline & $y_{t}, z_{t}$ & $(0.00,-0.723)$ & - & $\begin{array}{l}(0.00,- \\
0.722)\end{array}$ \\
\hline & $\begin{array}{l}\text { No. } \\
\text { elements }\end{array}$ & 200 & 1500 & 8 \\
\hline
\end{tabular}

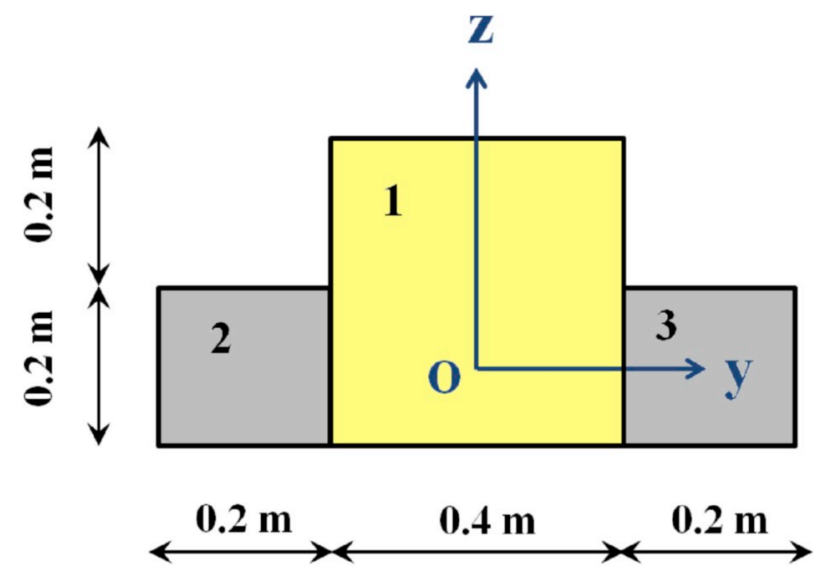

Fig. 7. Geometrical parameters of three-part composite cross-section.

The composite cross-section is discretized by using rectangular elements with size $1 \mathrm{~cm} \quad 1 \mathrm{~cm}$. The convergence mesh study shows that the chosen value for the size of elements is enough for a cross-sectional analysis. The torsional constant, warping constant as well as the coordinates of twist center of composite cross-section are given in Table 4.
Table 3

Mechanical properties of the three-part composite cross-section.

\begin{tabular}{llll}
\hline & Material 1 & Materials 2 & Materials 3 \\
\hline Young modulus (GPa) & 29 & 210 & 210 \\
Poisson's ratio & 0.3 & 0.3 & 0.3 \\
\hline
\end{tabular}

In this table, the present penalty-based finite element results are compared with boundary element results (Sapountzakis and Dourakopoulos, 2010), and finite element results by using linear triangle elements (Stoykov et al., 2015). It is observed that the results of the present model are in very good agreement with the results of other researchers.

Distribution of warping function $\varphi y, z$ over the three-part composite cross-section is shown in Fig. 8. In this figure, the results of the proposed formulation are compared with the results obtained by Stoykov et al. (2015). It is seen that the results of two different methods are in very good agreement quantitatively and qualitatively.

Remark. The above two examples approve the efficiency of the 2D penalty-based finite element formulation given in Section 3 for warping cross-sectional analysis.

\subsection{A cantilever sandwich beam under pure torsion}

A three-layer sandwich beam is analyzed in this section. The considered sandwich beam is a cantilever one and torque load of $\mathrm{T}$ $1000 \mathrm{kN} \mathrm{m}$ is applied at its free end. The length of beam is $\mathrm{L} \quad 1000 \mathrm{~mm}$ and its wide is b $400 \mathrm{~mm}$. The thickness of core layer is $h_{c} \quad 80 \mathrm{~mm}$ while the thickness of outer layers (skin) are $h_{s} \quad 10 \mathrm{~mm}$. The material properties of layers are as below:

Skin layers: $E_{1} \quad 3.0 \mathrm{GPa}, E_{2} \quad 0.8 \mathrm{GPa}, G_{12} \quad 1.05 \mathrm{GPa}, G_{22} \quad 1.05$ GPa, $V_{12} \quad V_{22} \quad 0.31$.

Core layer: E $\quad 60.0 \mathrm{MPa}, \mathrm{G} \quad 21.0 \mathrm{MPa}, V \quad 0.428$.

First, a convergence test was carried out by varying the number of axial and cross-sectional elements. The values of torsional rotation $\theta$ and warping parameter $\gamma$ at the free end of the sandwich beam are calculated with considering different number of axial and cross-sectional elements. The results are shown in Table 5 . It can be observed from this table that the values of $\theta$ and $\gamma$ are almost insensitive to the number of crosssectional elements. It is also seen that the warping parameter $\gamma$ has a high convergence velocity. Only 5 axial elements are enough for the evaluation of the warping parameter $\gamma$. Convergence velocity of the torsional rotation $\theta$ is lower and at least 20 axial elements is required. Based on numerical results of Table 5 , it is inferred that a mesh including 20 axial elements as well as 60 cross-sectional elements is enough for an accurate approximation of torsional rotation $\theta$ and warping parameter $\gamma$.

The sensitivity of in-plane normal displacement $(U)$ and shear stress components $\left(\sigma_{x y} \sigma_{x z}\right)$ against the number of cross-sectional elements is investigated in Table 6 . The results of this table are obtained by employing 20 axial elements. It is seen that the value of in-plane normal displacement is not sensitive with respect to cross-sectional meshing and

Table 4

Torsional constant, warping constant and the position of twist center for the three-part composite cross-section.

\begin{tabular}{llll}
\hline & $\begin{array}{l}\text { Boundary element }( \\
\text { Sapountzakis and } \\
\text { Dourakopoulos, 2010) }\end{array}$ & $\begin{array}{l}\text { Finite element }( \\
\text { Stoykov et al., } \\
\text { 2015) }\end{array}$ & Present \\
\hline$\widetilde{G J} / G_{1}\left(\mathrm{~m}^{4}\right)$ & $9.226 \mathrm{E}-03$ & $9.249 \mathrm{E}-03$ & $9.340 \mathrm{E}-$ \\
$\widetilde{E C_{w}} / E_{1}\left(\left(\mathrm{~m}^{6}\right)\right.$ & $1.755 \mathrm{E}-04$ & $1.754 \mathrm{E}-04$ & 03 \\
$y_{t}(\mathrm{~m})$ & 0.0 & 0.0 & $\begin{array}{l}1.747 \mathrm{E}- \\
04\end{array}$ \\
$z_{t}(\mathrm{~m})$ & $5.02 \mathrm{E}-02$ & $5.09 \mathrm{E}-02$ & 0.00 \\
& & & $5.14 \mathrm{E}-$ \\
\end{tabular}



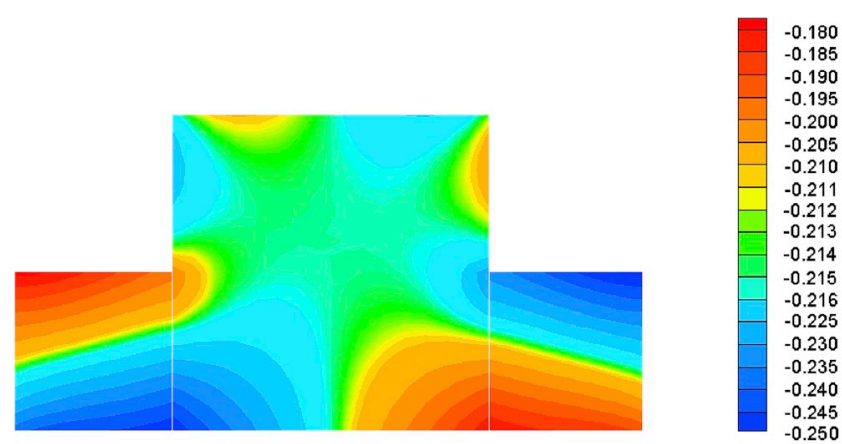

(a)

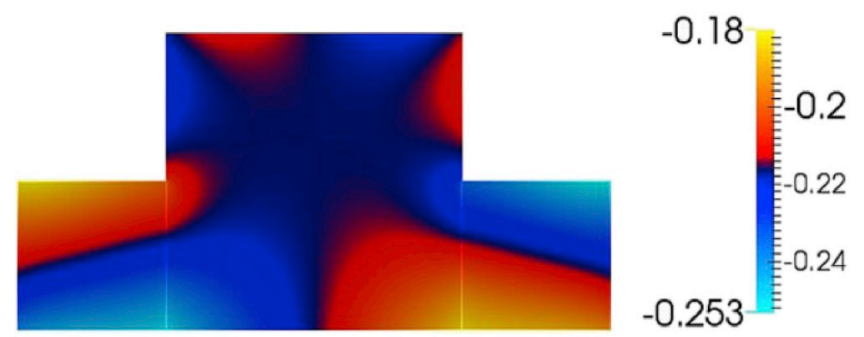

(b)

Fig. 8. Contour plot of warping function $\left(\mathrm{m}^{2}\right)$ for the three-part composite cross-section: (a) present, (b) Stoykov et al. (Stoykov et al., 2015).

it can be estimated accurately by using only 60 cross-sectional elements. As can be clearly observed from Table 6, the values of in-plane and transverse shear stresses are sensitive with respect to cross-sectional meshing and a relatively large number of cross-sectional elements are required to obtain the converged values. Based on mesh convergence results of Tables 5 and 6, one can conclude that a mesh including 20 axial elements as well as 600 cross-sectional elements is enough for the estimation of generalized displacement and stress components of the sandwich beam by using the present penalty-based multifiber finite element model.

Fig. 9 shows variations of transverse shear stress $\sigma_{x z}$ along the thickness and width of sandwich beam. Variations of in-plane stress $\sigma_{x y}$ along the width at the interface between core and skin layers are shown in Fig. 10. In these figures, results of present model are compared with 1D finite element results of Ganapathi et al. (1999) as well as 3D finite element solutions. It is noted that the formulation of Ganapathi and colleagues is based on a sinus shear deformation beam theory which includes torsional-warping effects. Ganapathi et al. (1999) obtained warping function from a 3D elasticity solution. Concerning the 3D finite element analysis, it is carried out by employing 112500 elements of 8-node brick type.

It can be observed that the results of the present penalty-based FE are in excellent agreement with reference solutions. Although the warping function was computed by enforcing the Saint-Venant's assumption $(\gamma$ $\left.d \theta_{x} / d x\right)$, it is seen from Fig. 9 that the continuity requirements of transverse shear stress at the interface between skin and core layers is satisfied. For more investigation of this issue, the variations of the unknown parameters $d \theta_{x} / d x$ and $\gamma$ along the length of the sandwich beam are depicted in Fig. 11. It is seen from this figure that $d \theta_{x} / d x$ and $\gamma$ approach each other, except the region near clamped support. Thus, one can conclude that the simplifying assumption $\gamma d \theta_{x} / d x$ used for obtaining the warping function (Section 3 ) is rational and acceptable.

Contour plot of in-plane normal displacement $U$ at free end of sandwich beam (i.e. $x \quad$ L) is shown in Fig. 12. In this figure, the contour plot of 3D finite element model is also given for the comparison purposes. Two sets of results are in very good agreement quantitatively and qualitatively. It can be noted from Fig. 12 that in-plane normal displacement $U$ varies anti-symmetrically about both width and thickness of beam. The maximum value of $U$ occurs along the edges of width at interface between core and skin layers. The maximum value of inplane normal displacement predicted by ABAQUS is $0.128 \mathrm{~mm}$. For the present penalty-based finite element model, this value is $0.129 \mathrm{~mm}$.

\subsection{Bending-torsion of a four-layer composite beam}

As a final example, a four layered composite beam with lamination scheme [0/90/90/0] is considered. Boundary and loading conditions of beam is shown in Fig. 13. The geometrical parameters considered for this example are given as L $\quad 2 \mathrm{~m}, \mathrm{~b} \quad 0.4 \mathrm{~m}$ and $\mathrm{h} \quad 0.1 \mathrm{~m}$. All layers have the same thickness. The material properties are assumed as follows (El Fatmi and Zenzri, 2002):

\section{$E_{1} \quad 137.6 \mathrm{GPa}, E_{2} \quad E_{2} \quad 14.448 \mathrm{GPa}, \mathrm{G} \quad 5.848 \mathrm{GPa}, \mathrm{v} \quad 0.21$}

For analysis by the proposed penalty-based finite element approach, the beam was divided into 40 equal sized multifiber elements. Concerning the cross-sectional meshing, the width and thickness of each layer were divided into 20 and 10 segments, respectively.

Contour plots of in-plane shear stress $\sigma_{x y}$ and transverse shear stress $\sigma_{x z}$ at a section with distance $\mathrm{L} / 4$ from the clamped support are shown in Figs. 14 and 15, respectively. In these figures, the results of present multifiber finite element model are compared with the results obtained from 3D finite element simulation (ABAQUS). It can be observed from Figs. 14-15 that a remarkable overall quantitative and qualitative

\section{Table 6}

Convergence test on the number of required cross-sectional elements for the evaluation of shear stresses (MPa) and in-plane normal displacement (mm).

\begin{tabular}{|c|c|c|c|c|c|}
\hline$m_{e}$ & $\tau_{x y} 1 m, 0$ & 0.05 & $\tau_{x z} 1 m, 0.2$ & 0.045 & $U 1 m, 0.2,0.04$ \\
\hline 60 & 2.354 & & 0.195 & & 0.129 \\
\hline 180 & 2.365 & & 0.351 & & 0.129 \\
\hline 300 & 2.375 & & 0.387 & & 0.129 \\
\hline 600 & 2.396 & & 0.398 & & 0.129 \\
\hline 900 & 2.398 & & 0.400 & & 0.129 \\
\hline 1200 & 2.398 & & 0.400 & & 0.129 \\
\hline
\end{tabular}

Table 5

Convergence test on $\theta(\mathrm{rad})$ and $\gamma(\mathrm{rad} / \mathrm{m})$ with increasing the number of axial and cross-sectional elements.

\begin{tabular}{|c|c|c|c|c|c|c|c|c|}
\hline \multirow{2}{*}{$\frac{n_{e}}{m_{e}}$} & \multicolumn{2}{|l|}{5} & \multicolumn{2}{|l|}{10} & \multicolumn{2}{|l|}{20} & \multicolumn{2}{|l|}{40} \\
\hline & $\begin{array}{ll}\theta & 10^{2}\end{array}$ & $\gamma \quad 10^{2}$ & $\begin{array}{ll}\theta & 10^{2}\end{array}$ & $\gamma \quad 10^{2}$ & $\begin{array}{ll}\theta & 10^{2}\end{array}$ & $\gamma \quad 10^{2}$ & $\begin{array}{ll}\theta & 10^{2}\end{array}$ & $\gamma \quad 10^{2}$ \\
\hline 3 & 3.468 & 3.905 & 3.701 & 3.906 & 3.759 & 3.906 & 3.766 & 3.906 \\
\hline 60 & 3.467 & 3.902 & 3.700 & 3.903 & 3.758 & 3.903 & 3.764 & 3.903 \\
\hline 180 & 3.465 & 3.895 & 3.698 & 3.895 & 3.756 & 3.895 & 3.762 & 3.895 \\
\hline 300 & 3.462 & 3.889 & 3.695 & 3.890 & 3.755 & 3.890 & 3.760 & 3.890 \\
\hline 600 & 3.462 & 3.889 & 3.695 & 3.890 & 3.755 & 3.890 & 3.760 & 3.890 \\
\hline
\end{tabular}




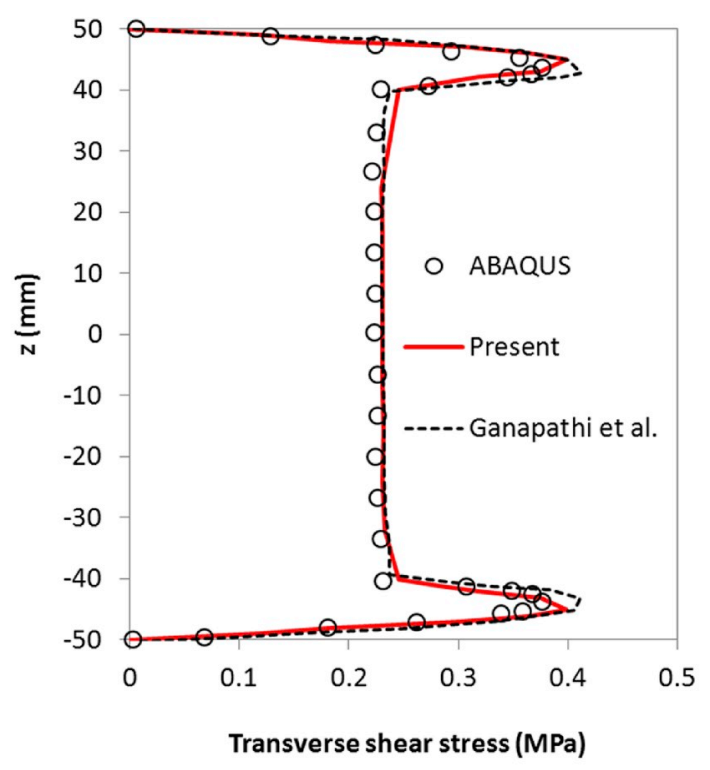

(a)

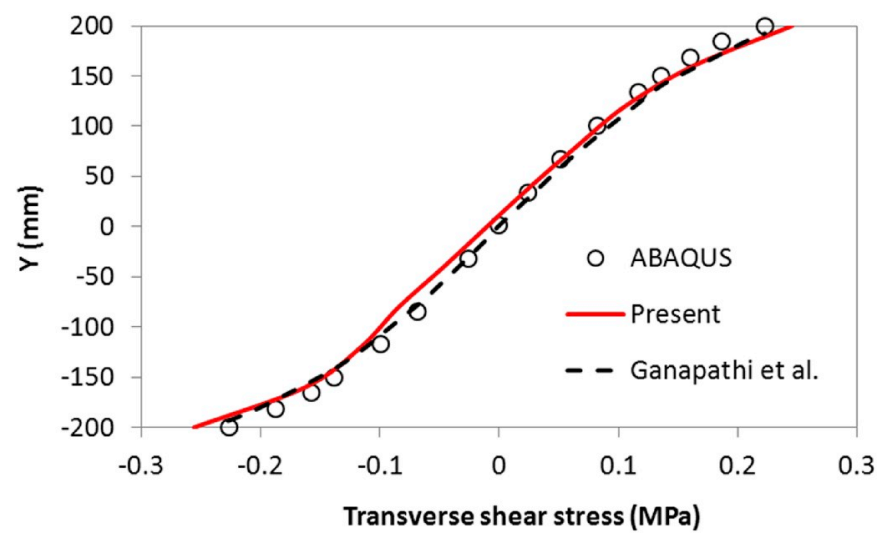

(b)

Fig. 9. Distributions of transverse shear stress $\sigma_{x z}$ : (a) along the thickness direction (x $1000 \mathrm{~mm}, \mathrm{y} \quad 200 \mathrm{~mm}$ ), (b) along the width direction (x 1000 $\mathrm{mm}, \mathrm{z} \quad 40 \mathrm{~mm})$.

agreement exists between the two sets of results. Note that 3D finite element simulation is carried out by employing $\begin{array}{lll}30 & 40 & 50 \text { elements }\end{array}$ of 8-node brick type. The number of degrees of freedom in the ABAQUS model is 194,463 while this value for the present multifiber finite element model is 3179.

It can be observed from Fig. 14 that the in-plane shear stress $\sigma_{x y}$ varies symmetrically about width while its distribution along the thickness is anti-symmetric. Similar to the 3D finite element solution, the in-plane shear stress computed by the present penalty-based multifiber finite element model satisfies the zero stress conditions at the edges of the width. In both present and 3D finite element models, maximum inplane shear stresses occur at the middle of the upper and lower surfaces. The predicted value of ABAQUS simulation for the maximum shear stress $\tau_{x y}$ at the considered section is $2.01 \mathrm{MPa}$. This value for the present multifiber model is $2.06 \mathrm{MPa}$ (error is about $2.5 \%$ ).

Fig. 15 shows that the distribution of transverse shear stress along the thickness direction is symmetric. Through-the-thickness variations of $\sigma_{x z}$ at two different sections are depicted in Fig. 16. It can be observed that the predicted transverse shear stress $\sigma_{x z}$ maintains its continuity along the thickness and fulfills the stress-free requirement on the upper and lower surfaces of the laminate. Due to the simultaneous effects of

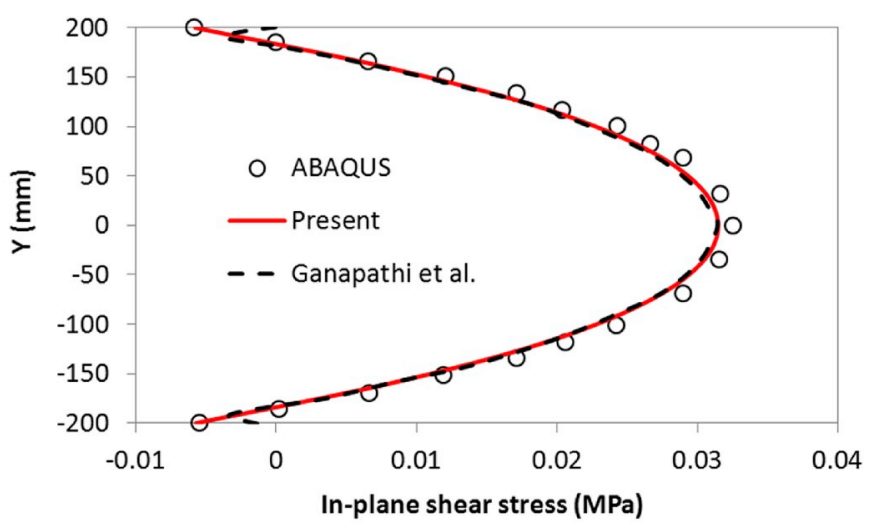

(a)

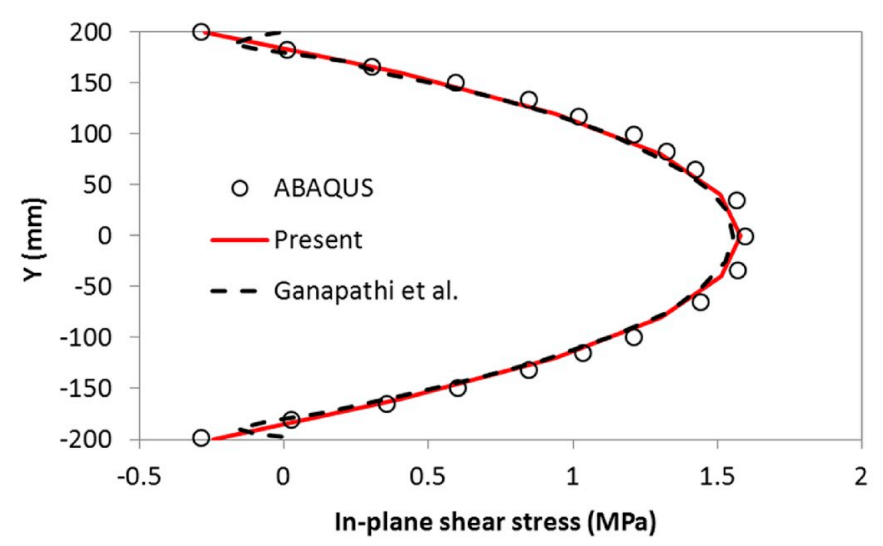

(b)

Fig. 10. Distributions of in-plane shear stress $\sigma_{x y}$ along the width direction at interface: (a) core (x $1000 \mathrm{~mm}, \mathrm{z} \quad 40 \mathrm{~mm}$ ), (b) skin (x $1000 \mathrm{~mm}, \mathrm{z}$ $40 \mathrm{~mm})$.

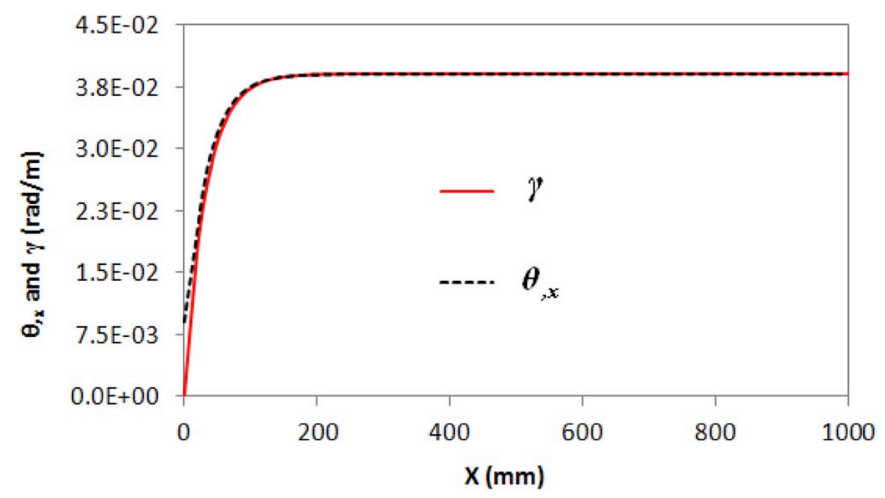

Fig. 11. Variations of $d \theta / d x$ and $\gamma$ along the length of sandwich beam.

bending and torsion actions, the transverse shear stress distribution does not vary anti-symmetrically along width. In case of pure torsion, distributions of transverse shear stress about width are anti-symmetrical. In both present and 3D finite element models, maximum of transverse stresses occur at the edges of the width. The maximum value of shear stress $\sigma_{x z}$ predicted by ABAQUS is $2.11 \mathrm{MPa}$ while that value is $2.00 \mathrm{MPa}$ for the present model. Note that the bending contribution of transverse shear stress $\sigma_{x z}$ is calculated directly from the constitutive equations. Although the calculation of bending shear stress by integrating the equilibrium equations leads to more accurate results, the direct estimation of the stresses from the constitutive equations is preferred for 


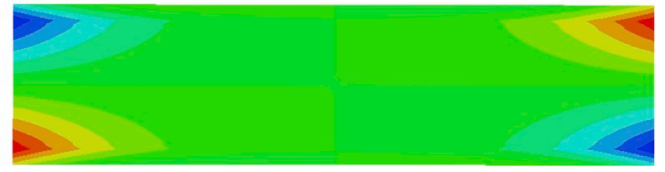

(a)

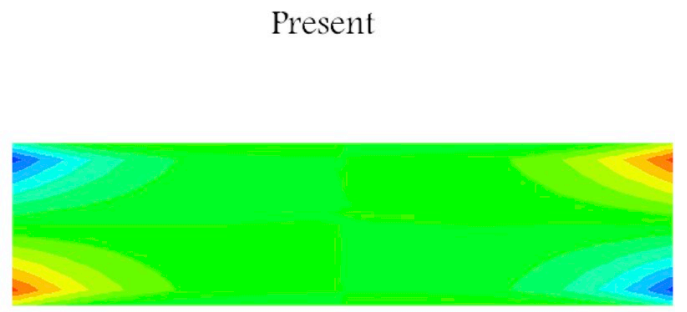

(b)

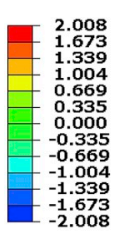

ABAQUS

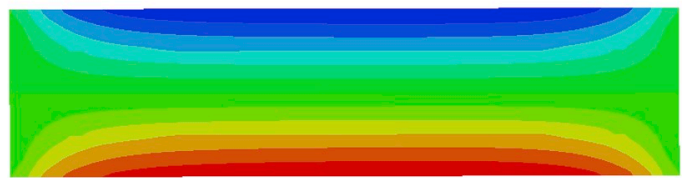

(a)

Present

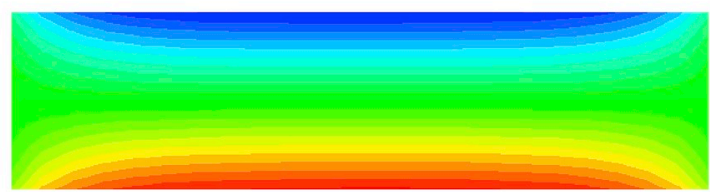

(b)

Fig. 14. Contour plot of in-plane shear stress $\sigma_{x y}(\mathrm{MPa})$ at $(\mathrm{L} / 4, \mathrm{y}, \mathrm{z})$ : (a) ABAQUS, (b) present.

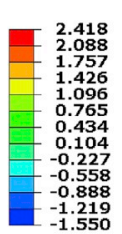

ABAQUS

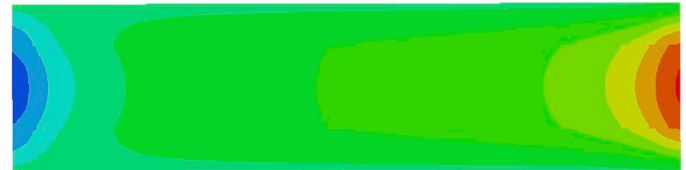

(a)

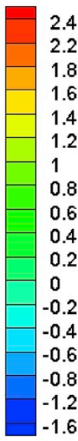

Present

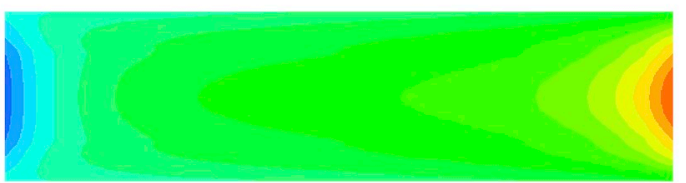

(b)

Fig. 15. Contour plot of transverse shear stress $\sigma_{x z}(\mathrm{MPa})$ at $(\mathrm{L} / 4, \mathrm{y}, \mathrm{z})$ : (a) ABAQUS, (b) present.

\section{Conclusions}

For the first time, an efficient and accurate penalty-based multifiber finite element model was developed for coupled bending and torsionalwarping analysis of laminated composite and sandwich beams. Displacement fields of composite beams were obtained by the 


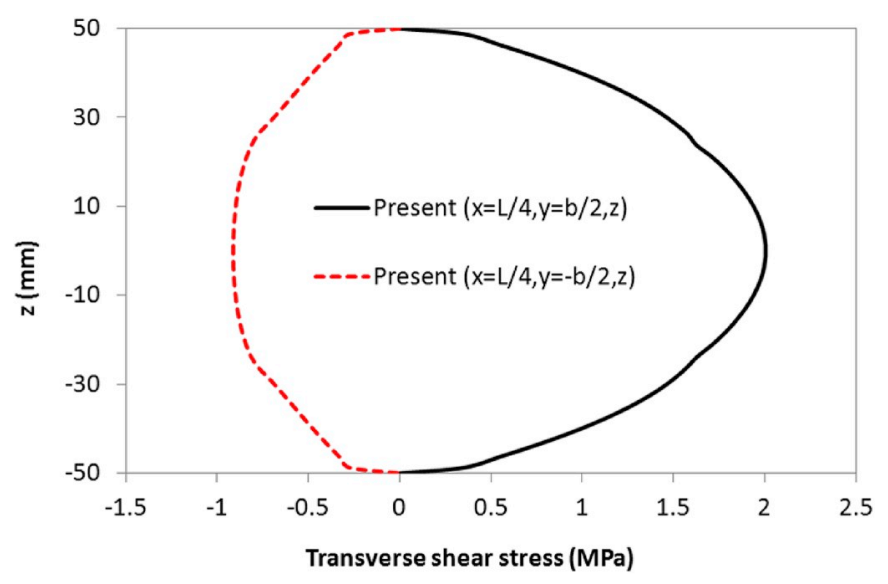

Fig. 16. Through-the-thickness variations of $\sigma_{x z}$ at two different sections.

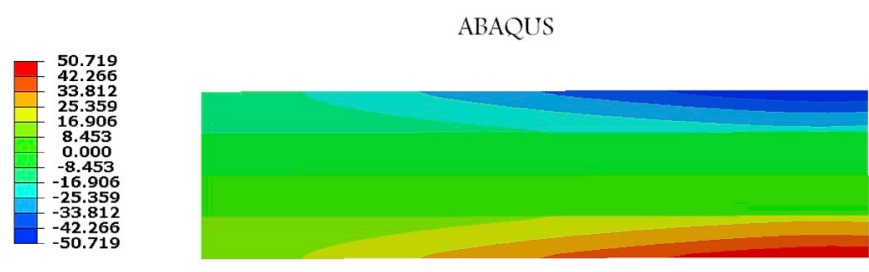

(a)

Present
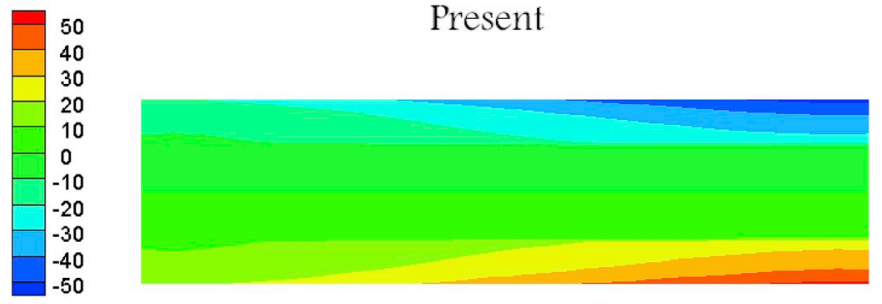

Fig. 17. Contour plot of in-plane normal stress $\sigma_{x x}$ at $(\mathrm{L} / 4, \mathrm{y}, \mathrm{z})$ : (a) ABAQUS, (b) present.

superimposition of displacement fields of composite beam due to axial extension, bending and torsion. For representing the displacement field of composite beam in pure bending, refined sinus shear deformation beam theory was used. Concerning the displacement field associated to torsion, restrained warping-torsion theory of Vlasov was employed.

A four-node full-compatible Hermitian element was employed to compute the warping function from the elliptic partial differential equation. To enforce the torsional-warping contribution of in-plane normal displacement and transverse shear stress at the layer interfaces, the penalty function approach was used. A three-node 1D multifiber finite element model was derived for approximation of unknown variables of displacement field. Finally, discrete form of governing equations were derived from the principle of minimum potential energy. Shear correction factor is not needed in the present formulation and traction-free conditions of shear stress components on exterior edges of beam is guaranteed. Moreover, the continuity conditions of transverse shear component of stress tensor as well as all displacement components are fulfilled at the interface between adjacent layers. Various examples of composite beams under combined transverse and torsional loads were analyzed to assess the present multifiber finite element model. Numerical results were compared with 3D finite element solutions, analytical and numerical results reported by other researchers. It was observed that the results of present model are in very good agreements with other reference solutions. Indeed, it was proved

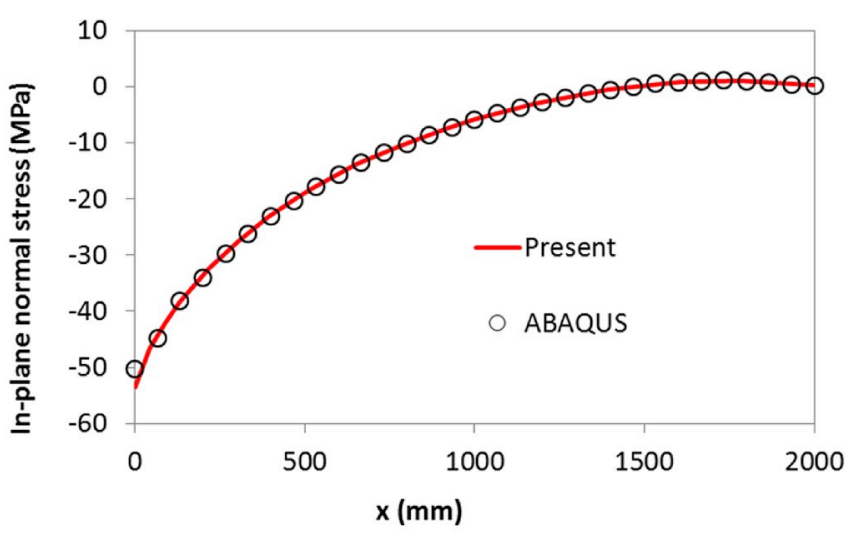

(a)

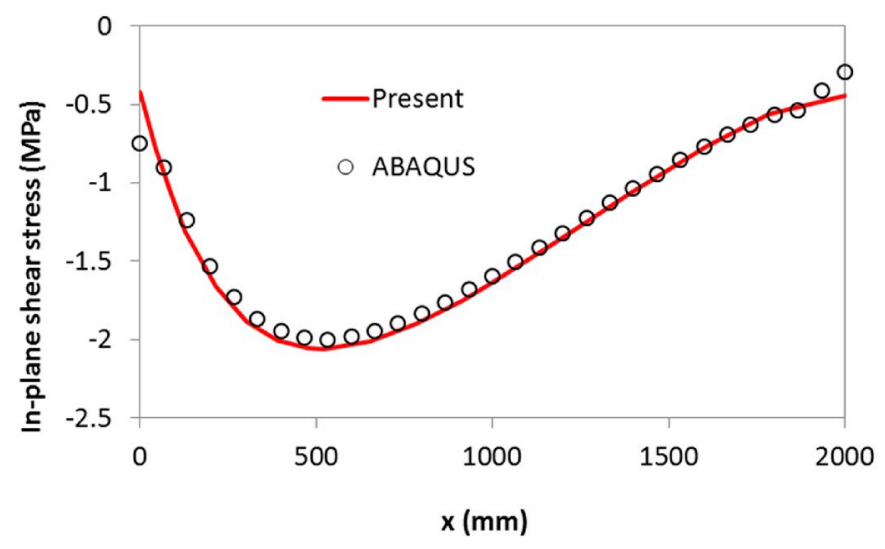

(b)

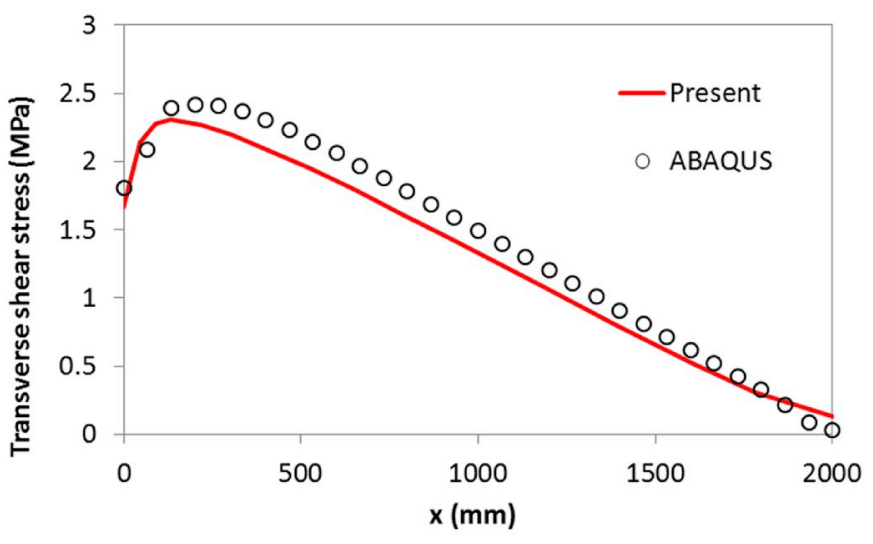

(c)

Fig. 18. Variations of stress components along the length of the cantilever beam: (a) $\sigma_{x x}\left(\mathrm{x}, \mathrm{y} \quad 200 \mathrm{~mm}, \mathrm{z} \quad 50 \mathrm{~mm}\right.$ ), (b) $\sigma_{x y}\left(\mathrm{x}, \mathrm{y} \quad 0, \mathrm{z} \quad 50 \mathrm{~mm}\right.$ ), (c) $\sigma_{x x}$ (x,y $200 \mathrm{~mm}, \mathrm{z} \quad 0)$.

that the present formulation can predict the quasi-3D responses of composite beams by using a relatively small number of degrees of freedom. The ratio of number of dofs of present model to the 3D finite element model is about $1 / 60$.

Future researches are toward the micromechanics-based progressive failure analysis of laminated composite beams by using the present multifiber finite element method.

\section{Declaration of competing interest}

The authors declared no potential conflicts of interest with respect to 


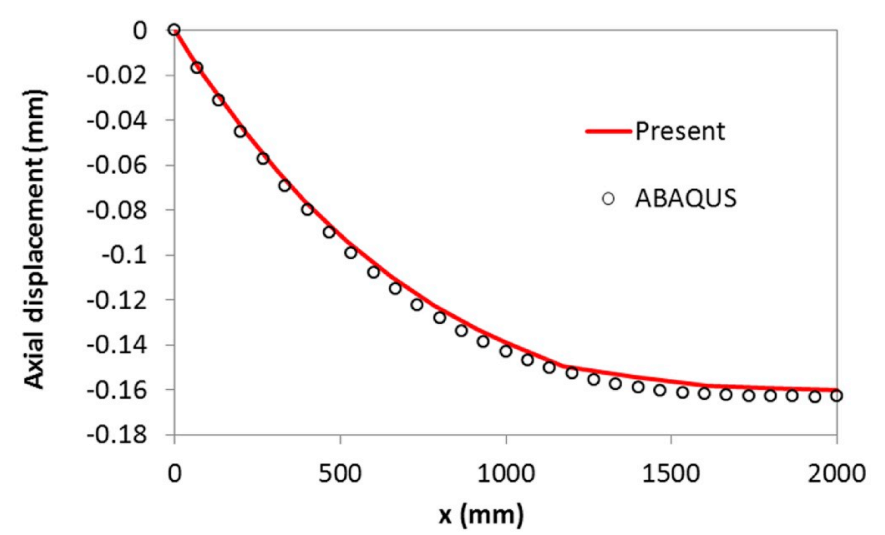

(a)

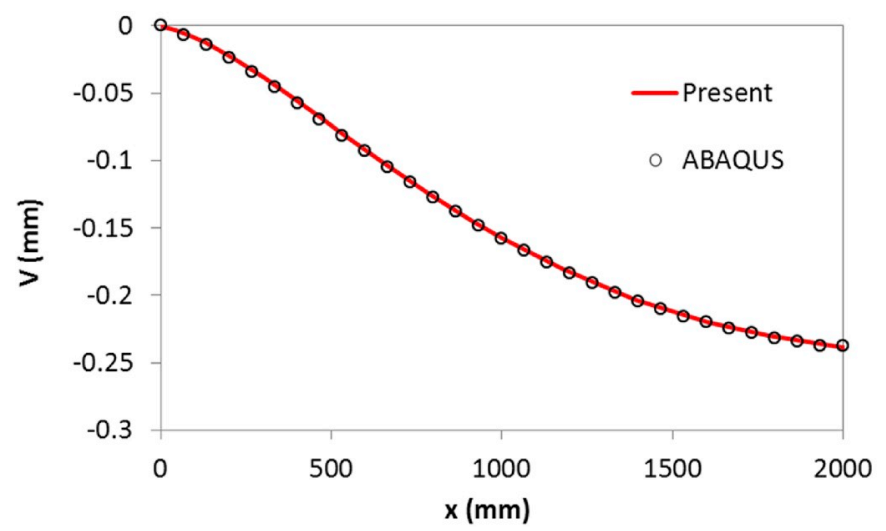

(b)

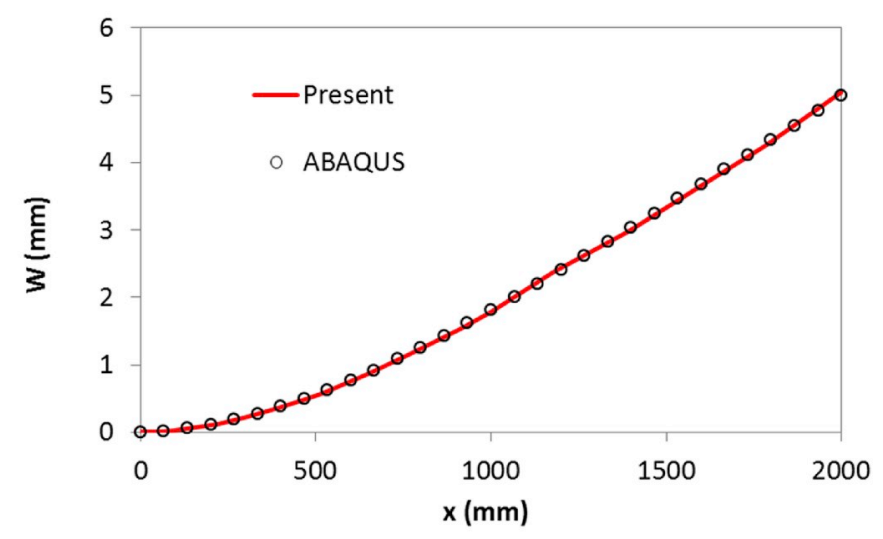

(c)

Fig. 19. Longitudinal variations of displacement components: (a) $U(x, y \quad 0, z$ h/2), (b) V(x,y 0,z h/2), (c) W(x,y 0,z h/2).

the research, authorship, and/or publication of this article.

\section{Appendix A}

The arrays of matrix $\mathbf{K}_{\varphi}$ can be obtained from the following relations:
$\mathbf{K}_{\phi}{ }_{i}^{k}{ }^{1} n_{\widehat{\phi}},{ }_{i}^{k}{ }^{1} n_{\widehat{\phi}} \quad p_{\phi}$
$\mathbf{K}_{\phi} \quad{ }_{i}^{k}{ }^{1} n_{\widehat{\phi}},{ }_{j}{ }_{j} n_{\phi} \quad p_{\phi} \quad k \quad 1,2, \ldots, N C \quad 1$
$\mathbf{K}_{\phi}{ }_{j}^{k} n_{\hat{\phi}},{ }_{i}{ }^{1} n_{\hat{\phi}} \quad p_{\phi} \quad i, j \quad 1,2, \ldots \ldots, n_{z_{k} 1}$
$\mathbf{K}_{\phi}{ }_{j}^{k} n_{\phi},{ }_{j}^{k} n_{\phi} \quad p_{\phi}$ 
where ${ }_{i}^{k}{ }^{1} n_{\widehat{\varphi}}$ and ${ }_{j}^{k} n_{\widehat{\varphi}}$ are the number of row in $\widehat{\boldsymbol{\Phi}}$ which correspond to $\widehat{\varphi}_{i}^{k}{ }^{1}$ and $\widehat{\varphi}_{j}^{k}$, respectively. The arrays of matrix $\mathbf{K}_{\varphi_{y}}$ can be obtained as below:

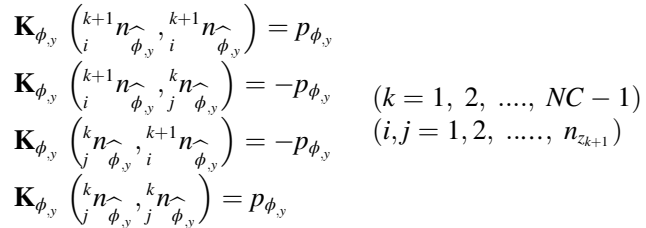

where ${ }_{i}^{k}{ }^{1} n_{\widehat{\varphi}_{y}}$ and ${ }_{j}^{k} n_{\widehat{\varphi}_{y}}$ are the number of row in $\widehat{\boldsymbol{\Phi}}$ which are corresponding to $\widehat{\varphi}_{i, y}^{k}{ }^{1}$ and $\widehat{\varphi}_{j, y}^{k}$, respectively. The arrays of matrix $\mathbf{K}_{\varphi_{y}}$ are given by:

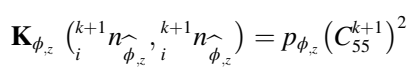

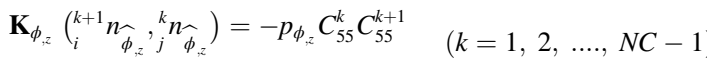

$\mathbf{K}_{\phi, z}{ }_{j}^{k} n_{\widehat{\phi}_{z,},{ }_{i}^{k}}{ }^{1} n_{\widehat{\phi}, z} \quad p_{\phi_{, z}} C_{55}^{k} C_{55}^{k}{ }^{1} \quad i, j \quad 1,2, \ldots ., n_{z k 1}$

$\mathbf{K}_{\phi, z}{ }_{j}^{k} n_{\widehat{\phi}_{, z}},{ }_{j}^{k} n_{\widehat{\phi}_{, z}} \quad p_{\phi_{, z}} C_{55}^{k}{ }^{2}$

where ${ }_{i}^{k}{ }^{1} n_{\widehat{\varphi}_{, z}}$ and ${ }_{j}^{k} n_{\widehat{\varphi}_{y, z}}$ are the number of row in $\widehat{\boldsymbol{\Phi}}$ which are corresponding to $\widehat{\varphi}_{i, z}^{k}{ }^{1}$ and $\widehat{\varphi}_{j, z}^{k}$, respectively. The arrays of $\mathbf{Q}_{\varphi_{, z}}$, which is a vector with size $n$ 1, are obtained from the following relations:

$\begin{array}{lllllllll}\mathbf{Q}_{\phi_{z}}{ }^{k} & i & 1 \\ n_{\phi_{, z}}, 1 & p_{\phi_{, z}} C_{55}^{k}{ }^{1} C_{55}^{k} & C_{55}^{k}{ }^{1} y_{i} & k & 1,2, \ldots, N C & 1\end{array}$

$\mathbf{Q}_{\phi_{, z}}{ }_{j}^{k} n_{\hat{\phi}_{, z}}, 1 \quad p_{\phi_{, z}} C_{55}^{k} C_{55}^{k} \quad C_{55}^{k}{ }^{1} y_{i} \quad i, j \quad 1,2, \ldots ., n_{z k 1}$

Based on numerical experience, the value of penalty parameters $p_{\varphi}, p_{\varphi_{y}}$ and $p_{\varphi_{, z}}$ were assumed $10^{4}$ times of the largest absolute value of entry of matrix $\mathbf{K}$.

\section{Appendix B}

The vectors of degrees of freedom of the fiber elements are defined as:

$$
\begin{aligned}
& \widehat{\mathbf{u}}_{\mathbf{u}}^{\mathbf{e}} \quad\left\{\begin{array}{ll}
\widehat{u}_{1} & \widehat{u}_{2}
\end{array}\right\}^{T} \\
& \begin{array}{lllll}
\widehat{\mathbf{u}}_{\mathbf{w}}^{\mathbf{e}} & \widehat{w}_{1} & \widehat{w}_{1, x} & \widehat{w}_{2} & \widehat{w}_{2, x} T
\end{array} \\
& \begin{array}{ccccc}
\widehat{\mathbf{u}}_{\mathbf{v}}^{\mathbf{e}} & \widehat{v}_{1} & \widehat{v}_{1, x} & \widehat{v}_{2} & \widehat{v}_{2, x}{ }^{T}
\end{array} \\
& \begin{array}{llll}
\widehat{\mathbf{u}}_{\boldsymbol{\theta}_{\mathbf{y}}}^{\mathbf{e}} & \widehat{\theta}_{y 1} & \widehat{\theta}_{y 3} & \widehat{\theta}_{y 2}{ }^{T}
\end{array}
\end{aligned}
$$

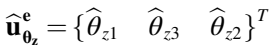

$$
\begin{aligned}
& \widehat{\mathbf{u}}_{\mathbf{u}_{\text {loc }}}^{\mathbf{e}} \quad \widehat{u}_{1}^{l o c} \widehat{u}_{3}^{l o c} \quad \widehat{u}_{2}^{l o c} \quad T \\
& \widehat{\mathbf{u}}_{\boldsymbol{\theta}_{\mathbf{x}}}^{\mathbf{e}} \quad\left\{\begin{array}{ll}
\widehat{\theta}_{x 1} & \widehat{\theta}_{x 2}
\end{array}\right\}^{T} \\
& \widehat{\mathbf{u}}_{u}^{\mathbf{e}} \quad\left\{\begin{array}{ll}
\widehat{\gamma}_{1} & \widehat{\gamma}_{2}
\end{array}\right\}^{T}
\end{aligned}
$$

Interpolating matrices $\mathbf{N}_{\mathbf{L L}}, \mathbf{N}_{\mathbf{H}}$ and $\mathbf{N}_{\mathbf{Q L}}$ are defined as:

$$
\begin{aligned}
& \mathbf{N}_{\mathbf{L L}} \quad\left\{\begin{array}{llll}
1 & r / 2 & 1 & r / 2
\end{array}\right\}^{T} \\
& \mathbf{N}_{\mathbf{H}} \quad\left\{\begin{array}{llllllll}
H_{01} & r & H_{11} & r & H_{02} & r & H_{12} & r
\end{array}\right\}^{T}
\end{aligned}
$$

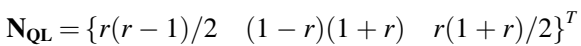

\section{References} Beheshti-Aval, S.B., Lezgy-Nazargah, M., 2010. A finite element model for composite
beams with piezoelectric layers using a sinus model. J. Mech. 26 (2), 249-258.
Beheshti-Aval, S.B., Lezgy-Nazargah, M., Vidal, P., Polit, O., 2011. A refined sinus finite
element model for the analysis of piezoelectric-laminated beams. Int. J. Mater. Syst.
Struct. 22 (3), 203-219.
Beheshti-Aval, S.B., Lezgy-Nazargah, M., 2012. A new coupled refined high-order globallocal theory and finite element model for electromechanical response of smart laminated/sandwich beams. Arch. Appl. Mech. 82 (12), 1709-1752.

Carrera, E., 2000. Single-layer vs multi-layers plate modeling on the basis of Reissner's mixed theorem. AIAA J. 38, 342-343.

Carrera, E., 2003. Historical review of Zig-Zag theories for multilayered plates and shells. Appl. Mech. Rev. 56 (3), 287-308.

Connor, J.J., 1976. Analysis of Structural Member Systems. Ronald Press, US. 
Demakos, C.B., 2003. Stress fields in fiber reinforced laminate beams due to bending and torsion moments. J. Reinf. Plast. Compos. 22 (5), 399-418.

El Fatmi, R., Zenzri, H., 2002. On the structural behavior and the Saint Venant solution in the exact beam theory, Application to laminated composite beams. Comput. Struct. $80,1441-1456$

El Fatmi, R., Zenzri, H., 2004. A numerical method for the exact elastic beam theory. Applications to homogeneous and composite beams. Int. J. Solids Struct. 41, 2521-2537.

El Fatmi, R., 2007. Non-uniform warping including the effects of torsion and shear forces. Part I: a general beam theory. Int. J. Solids Struct. 44, 5912-5929.

El Fatmi, R., Ghazouani, N., 2011. Higher order composite beam theory built on SaintVenant's solution. Part-I: theoretical developments. Compos. Struct. 93, 557-566.

Ganapathi, M., Patel, B.P., Polit, O., Touratier, M., 1999. A C ${ }^{1}$ finite element including transverse shear and torsion warping for rectangular sandwich beams. Int. J. Numer. Methods Eng. 45, 47-75.

Icardi, U., 2001a. Higher-order zig-zag model for analysis of thick composite beams with inclusion of transverse normal stress and sublaminates approximations. Compos. Part B 32, 343-354.

Icardi, U., 2001b. A three-dimensional zig-zag theory for analysis of thick laminated beams. Compos. Struct. 52, 123-135.

Katariya, P.V., Hirwani, C.K., Panda, S.K., 2019. Geometrically nonlinear deflection and stress analysis of skew sandwich shell panel using higher-order theory. Eng. Comput. 35 (2), 467-485.

Katariya, P.V., Panda, S.K., 2019. Numerical evaluation of transient deflection and frequency responses of sandwich shell structure using higher order theory and different mechanical loadings. Eng. Comput. 35 (3), 1009-1026.

Lezgy-Nazargah, M., Shariyat, M., Beheshti-Aval, S.B., 2011. A refined high-order globallocal theory for finite element bending and vibration analyses of the laminated composite beams. Acta Mech. 217, 219-242.

Lezgy-Nazargah, M., Vidal, P., Polit, O., 2015. NURBS-based isogeometric analysis of laminated composite beams using refined sinus model. Eur. J. Mech. A Solid. 53, $34-47$.
Lezgy-Nazargah, M., 2017. Assessment of refined high-order global-local theory for progressive failure analysis of laminated composite beams. Acta Mech. 228 (5), 1923-1940.

Reddy, J.N., 2004. Mechanics of Laminated Composite Plates and Shells: Theory and Analysis, second ed. CRC Press, Boca Raton.

Santiuste, C., Sanchez-Saez, S., Barbero, E., 2008. Dynamic analysis of bending-torsion coupled composite beams using the Flexibility Influence Function Method. Int. J. Mech. Sci. 50, 1611-1618.

Sapountzakis, E.J., Dourakopoulos, J.A., 2010. Shear deformation effect in flexuraltorsional vibrations of composite beams by boundary element method (BEM). J. Vib. Control 16 (12), 1763-1789.

Savoia, M., Tullini, N., 1993. Torsional response of inhomogeneous and multilayered composite beams. Compos. Struct. 25, 587-594.

Saygun, A., Omurtag, M.H., Orakdogen, E., Girgin, K., Kucukarslan, S., Darilmaz, K., 2007. A simplified solution of the torsional rigidity of the composite beams by using FEM. Adv. Struct. Eng. 10 (5), 467-473.

Shariyat, M., 2010. A generalized global-local high-order theory for bending and vibration analyses of sandwich plates subjected to thermo-mechanical loads. Int. J. Mech. Sci. 52, 495-514.

Stoykov, S., Manoach, E., Margenov, S., 2015. An efficient 3D numerical beam model based on cross sectional analysis and Ritz approximations. Z. Angew. Math. Mech.: Z. Angew. Math. Mech. 1-22. https://doi.org/10.1002/zamm.201400139.

Swanson, S.R., 1998. Torsion of laminated rectangular rods. Compos. Struct. 42, 23-31.

Timoshenko, S.P., Goodier, J.N., 1970. Theory of Elasticity. McGraw-Hill, New York.

Vlasov, V.Z., 1959. Thin-Walled Elastic Bars (in Russian). Fizmatgiz, Moscow.

Vidal, P., Polit, O., 2008. A family of sinus finite elements for the analysis of rectangular laminated beams. Compos. Struct. 84, 56-72.

Wunderlich, W., Pilkey, W.D., 2003. Mechanics of Structures: Variational and Computational Methods. CRC Press. 